Historic, Archive Document

Do not assume content reflects current scientific knowledge, policies, or practices 

1955.

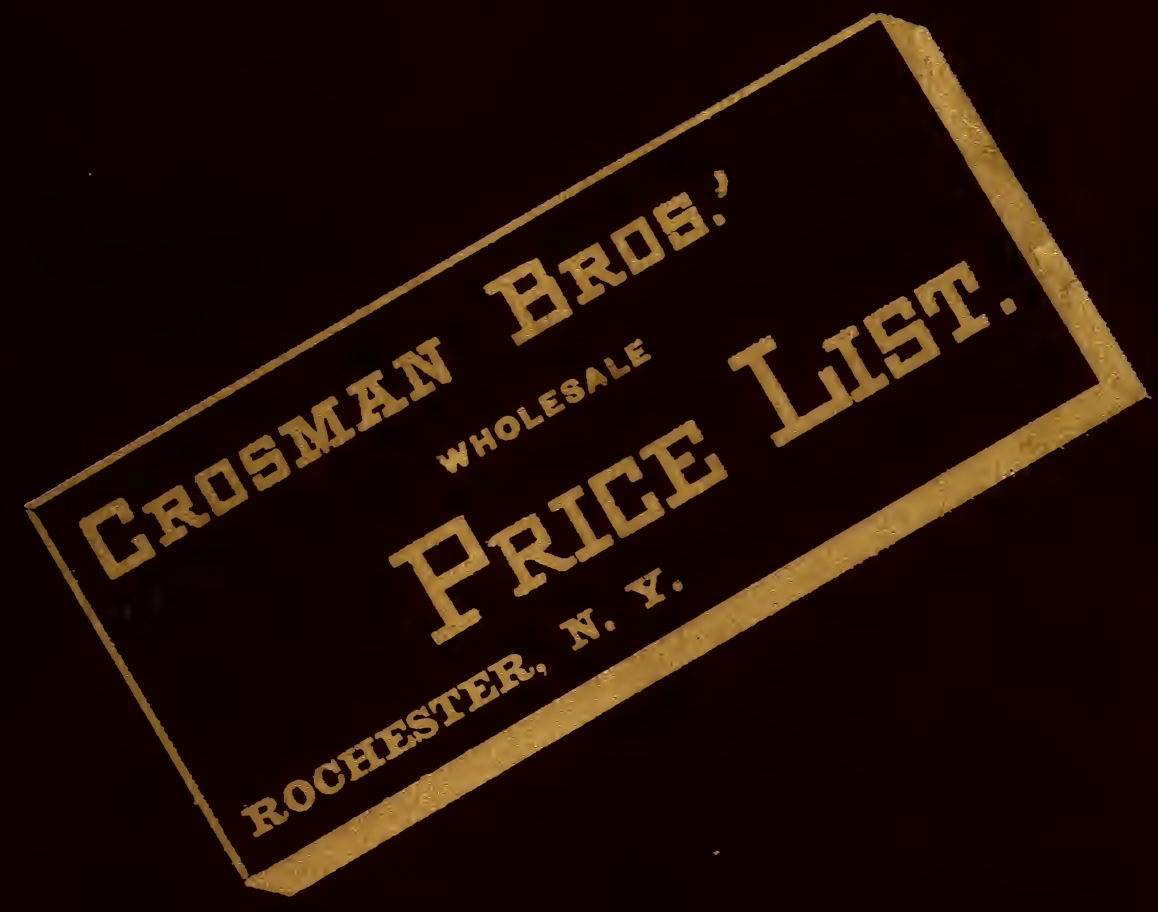

THIS LIST CARCFLS AIT PREVIOUS OFTERS. . . 


\section{VALUABLE TABLES.}

\section{Quantity of Seed Requisite to Produce a Given Number of Plants and Sow an Acre of Ground.}

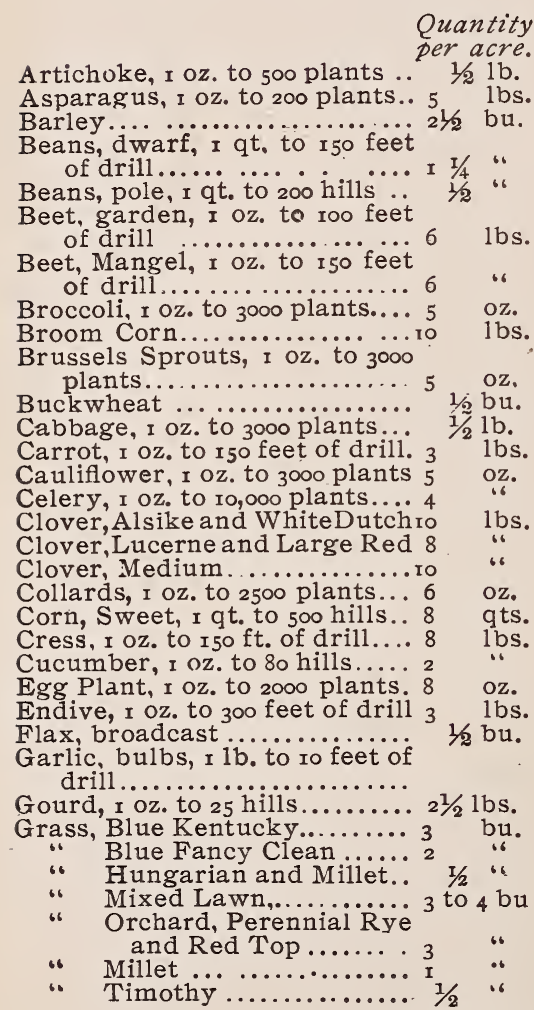

Quantity per acre.

Hemp................ $1 / 2$ bu.

Kale, I oz. to 3000 plants ....... 4 oz.

Kohl Rabi, r oz. to 200 feet of drill.................. $11 / 2$ lbs:

Leek, I oz. to 200 feet of drili 4

Lettuce, I oz. to 250 feet of drili 3

Martynia, I oz. to $50 \mathrm{ft}$. of drill ro

Melon, Musk, I oz. to roo hills.. 2

Melon, Water, 1 oz, to 25 hills.. 4

Nasturtium, I oz. to 50 feet of drill.................. ro no "

Oats.............................

Onion Seed, r oz. to 200 feet of drill....................

Onion Seed, for sets..............

Onion Sets, 1 qt. to $20 \mathrm{ft}$. of drill 8

列

Parsley, $\mathrm{x}$ oz. to 250 feet of drill 8

Peas, garden, 1 qt. to roo feet of drill

bu.

Peas, field ....................... ${ }_{21}^{1 \%}$

Pepper, I oz, to r 500 plants.... 4 Qz.

Potatoes.................

Pumpkin, I qt. to 3 () hills...... 4 qts.

Radish, I oz. to I50 feet of drili 8 lbs.

Rye... ...,.............. I1/6 bu.

Salsify, i oz. to 60 feet of drili. 8 : $1 \mathrm{bs}$,

Spinage, I oz. to I $50 \mathrm{ft}$. of drill. Io

Summer Savory, r oz. to $500 \mathrm{ft}$. of drill................. 2

Squash, Summer, I oz. to 40 hills.................... 2

Squash, Winter, 1 oz. to ro hills 3

Tomato, I oz. to 3000 plants .. 3

Tobacco, I oz. to 5000 plants... 2

Turnip, I oz. to 250 feet of drili 1

wheat................... 2 to 2 bu

\section{Number of Plants or Trees to the Acre at Given Distances.}

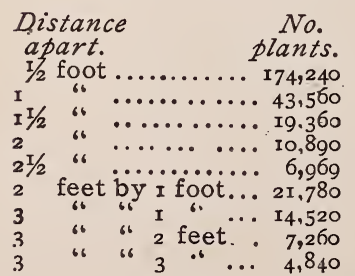$$
\begin{aligned}
& D \\
& 4 \\
& 4 \\
& 4 \\
& 4 \\
& 5 \\
& 6 \\
& 7 \\
& 8 \\
& 9
\end{aligned}
$$

Distance

apart.

No. plants.

4 " 2 feet..... 5,444

4 " 4 " 3 " 3 .... 3,629

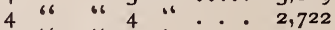

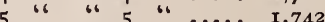

6 "

6
6 $\ldots \ldots \ldots \ldots$

1,2 IO

889

(................

680

573

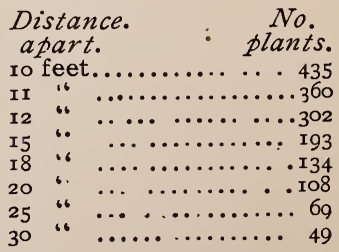


1840.

1895

ESTABLISHED FIFTY-FIVE YEARS.

\section{Wholesale Price List}

- OF-

Garden, Flower, Agricultural,

\section{Grass and Herb}

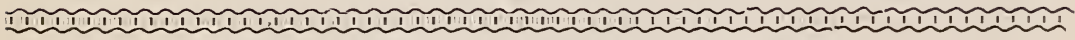
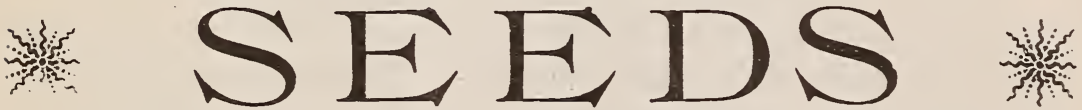

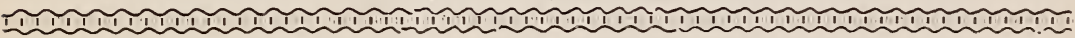

OFFERED BY

\section{CROSMAN BROTHERS,}

Growers, Importers of and Dealers in Seeds.

Wholesale Office and Seed House, 503 Monroe Ave.,

Retailstore, 23 South Clinton Street,

Greenhouses and trial Grounds, laburnum Crescent

ROCHESTER, N. Y.

Canadian Branehes: Coburgh and Wellington, Ont 


\section{TO OUR PATRONS.}

Rochester, N. Y., Jan. Ist, I895.

We thank our many friends and the trade generally for the liberal patronage bestowed on us in this department the past season, and in presenting you at this time with our Annual Wholesale Price List for 1895, to advise you that in consequence of the late rainy spring and long continued drouth both in this country and in Europe, that many seed crops have suffered severely, consequently short crop and slightly advanced prices on a few sorts.

An active and advancing market is looked for, but we are in hopes to be able to fill all orders intrusted to our care with promptness and dispatch.

We will try to adhere as closely as possible to the prices given, but wish it understood that we may have to advance on some varieties, but not unless compelled to do so.

We wish to call your attention to the fact that all our seeds are grown from the best selected stocks, and only by skilled and experienced growers, and consumers need only give them a trial to be convinced of their superior qualities.

We wish it particularly understood that we do not warrant them, nor do we hold ourselves in any way responsible for the crop. If the purchaser does not accept the seeds on these terms, they must be returned at once, carriage free.

A proportionately higher rate will be charged for quantities smaller than quoted, except that twenty-five pounds and over will be invoiced at the hundred-pound rate, and one-half bushel at bushel rate. 


\section{TERMS.}

Net Cash on June Ist, I895, without regard to the day of purchase; or a discount of one per cent. a month will be allowed on payments made before June ist. Goods purchased after June Ist, Net Cash October Ist; and after October Ist, Net Cash January Ist, following. Parties unknown to us wishing to order seeds, will be required to furnish satisfactory references, otherwise goods will be sent C. O. D.

Onion Sets, Potatoes, Grass, Grain, Fodder Corn and Clover Seed, are sold only for net cash.

Terms of Cash.-We will allow a discount of Five per Cent. on all orders accompanied by a cash remittance.

\section{SHIPPING INSTRUCTIONS.}

Shipping instructions should be sent with each order, as to the mode of shipment. When no such instructions are given, the goods will be forwarded by the cheapest and most direct route known to us. All shipments are made in good order, as shown by Bill of Lading, thus exempting us from any claims for damages, miscarriage, delays or loss.

\section{PACKAGES.}

Bags, barrels, boxes, crates, etc., will be charged only at cost price. No charge for cartage or packing. Purchasers will pay their own freight.

\section{NOTICE.}

While we exercise the greatest care to have all seeds pure and reliable, it is hereby mutually agreed between ourselves and the purchaser that we do not warrant the same, and are not in any respect liable or responsible for the seeds sold by us, or for any loss or damage arising from any failure thereof in any respect. If the purchaser does not accept the seeds on these terms, they must be returned at once, carriage free. 


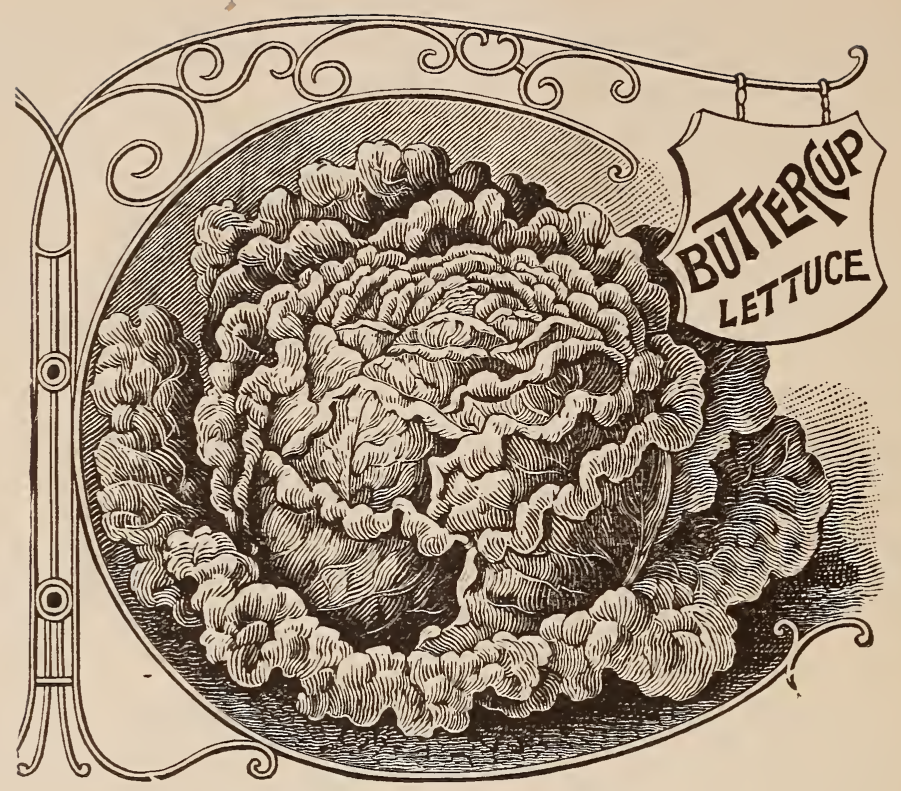

(See page I2.)

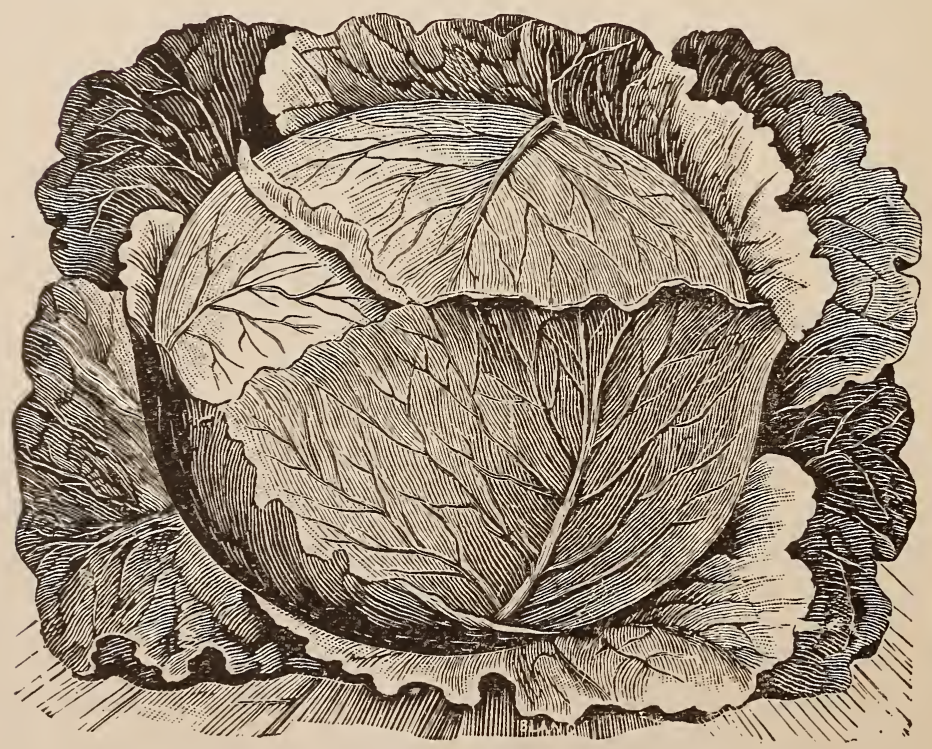

CABBAGE-PREMIUM FLAT DUTCH.

(See page 7.) 


\section{CROSMAN BROTHERS}

\section{WHOLESALE PRICE LIST \\ FOR 1895 .}

A Telegraphic Cipher is attached to each article, which will enable orders to be sent by wire at comparatively little expense. In dispatching, use the word Freight, Express or Mail, to designate how to send. For example :

Freight, two Acacia :-means send by Freight two bushels Early Dwarf Black Wax Beans ; or, Beet, etc.

Express, twenty Alarm:-means send by Express twenty pounds Eclipse

\section{Garden and Vegetable Seeds.}

\section{ARTICHOKE.}

Telegraph Cipher.

Green Globe.
Per $1 \mathrm{~b}$. $\$ 250$

ASPARAGUS.

Columbia Mammoth White............Abase I 25

Conover's Colossal....................Abandon I

Palmetto .......................Abate 35

Barr's Mammoth...................Aback 30

Roots of Colossal (two years old)...........Abber 4 oo

" " (one year old).......... Abbott 300

" of Palmetto (two years old)..........Abduct 450

" " (one year old)..........Ablaze 350

" of Columbia (one year old)..........Able 7 o

\section{BEANS, DWARF or SNAP.}

YELLOW PODDED VARIETIES.

Early Dwarf Black Wax, or butter.........Acacia 400

Prolific Black Wax...........Acantha 400

Challenge Black Wax.........Acarus 425

Golden Wax...............Accent 350

Improved Golden. Wax.........Accentor 350

Black-Eyed Wax ...........Accord 4 o०

Red Flagolet Wax...........Ache 425

Golden-Eyed Wax..........Acme 350

Wardwell's Kidney Wax......Ace 4 o०

“ Crystal White Wax............Across 400

Yosemite Mammoth Wax..............Active 8 o 
GREEN PODDED VARIETIES.

Improved Early Red Valentine (round pod). Adapt $\$ 275$

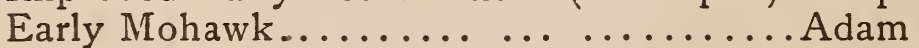

"Yellow Six Weeks (long)..........Adage

" China Red Eye...............Adore

" Emperor William..............Adolph

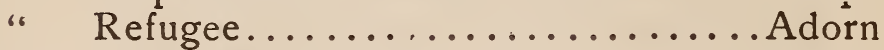

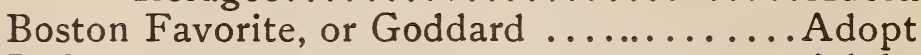

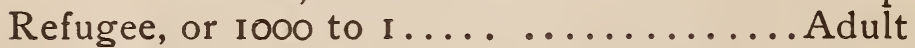

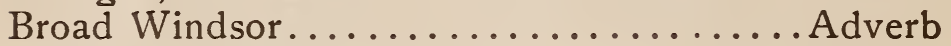

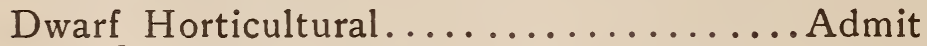

Improved Red Kidney................Advance

Royal Dwarf, or White Kidney..........Affair 300

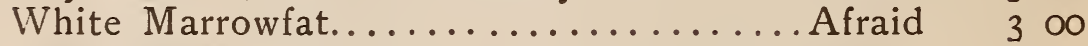

White Mediums...................After 250

Boston.Small Pea Bean................................. 300

Henderson's Dwarf Lima...............Afresh 525

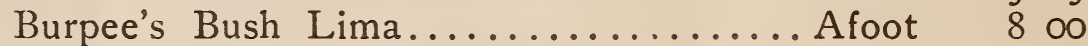

Gunkler, or New Prolific Pickler..........Africos 600

\section{BEANS, POLE or RUNNING.}

Dreer's New Golden Cluster Wax........Agast

German Black Wax (pole).............Again

Horticultural, or Speckled Cranberry......Age

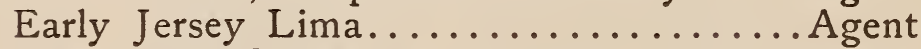

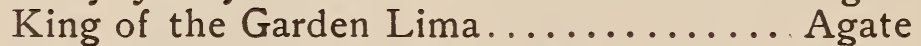

Large White Lima, Extra size........... Agree

300

275

275

400

300

275

275

400

300

300

.

600

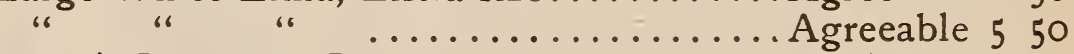

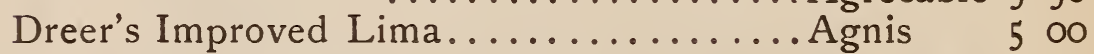

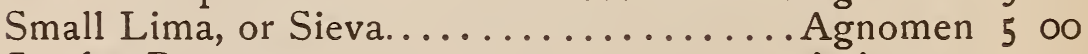

Scarlet Runners.......................................... 500

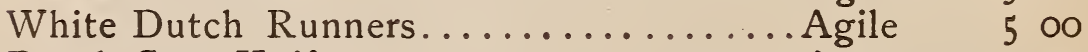

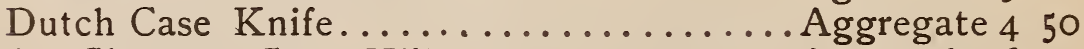

Cut Short, or Corn Hill.............Aground 6 oo

\section{BEET.}

Tel. Cipher.
Alarm

Dark Red Egyptian Turnip.......Albion

Edmond's Blood Turnip.........Albert

Extra Early Turnip Bassano.... Albany

Early Blood Turnip............Album

Bastian's Early Blood Turnip...AAlder

Lentz's

66

،... Aldine

$1001 \mathrm{bs}$.

Per $1 b$.

$\$ 2500$

30

2500

30

$2500 \quad 30$

$2000 \quad 25$

$2000 \quad 25$

$2000 \quad 25$

$2500 \quad 30$ 


\section{BEET-Continued.}

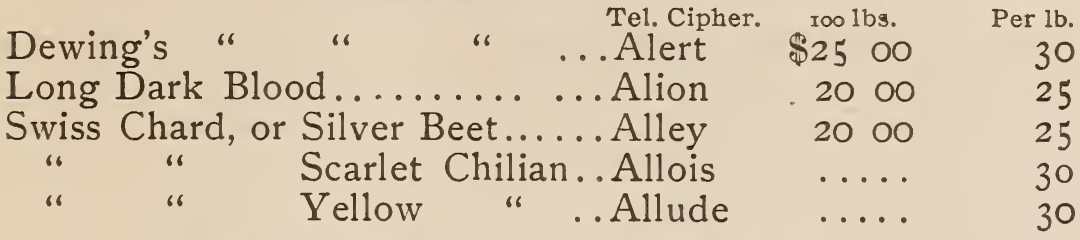

\section{SUGAR BEET AND MANGEL.}

Orange Globe................Amazon \$12 00 \$ 15

Prize Orange Globe............Amass 1500 I8

Mammoth Long Red...........Amber $\quad$ I2 $00 \quad$ I 5

Norbiton Giant Long Red .......Ambition I 2 oo . I 5

Yellow Ovoid (Intermediate).... Amend I2 00 I 5

Red Ovoid (Intermediate).......A Amateur I2 $00 \quad$ I5

Golden Tankard (Intermediate). Amiable I 5 oo I8

French White Sugar (Red Top). Amount I2 00 I5

Lane's Imperial White Sugar...A Amuse 12 oo 15

BROCCOLI.

Early Purple Cape............Amaze $\quad 200$

Early White Cape............Amid $25 \mathrm{C}$

\section{BRUSSELS SPROUTS.}

Brussels Sprouts, Tall Extra, select stock...Amity I oo " " Dwarf Improved........Amidst I IO

CABBAGE.

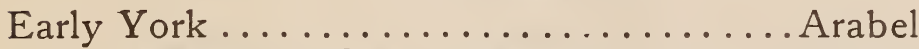

" Jersey Wakefield (American).........Arbor I 25

" " " (Imported).........Arch 75

"Winningstadt....................... 85

“ Etampes (Extra Early)........... Archery 60

Extra Early Express.................. Archer 70

Early Flat Dutch.......................... 75

All Head Early.....................Ardor I o

Henderson's Early Summer (American).... Area I 25

90

All Seasons (Imported) ................Aregood

90

" " (American) ...............Argue I 25

Filderkraut ....................... I oo

Premium Flat Dutch (Imported) ..........Armor $\quad 75$

" " " (American)............Army I OO

Large, Late Drumhead (Imported)........... Aroma
"

(American)........ Arsenal 


\section{CABBAGE-Continued.}

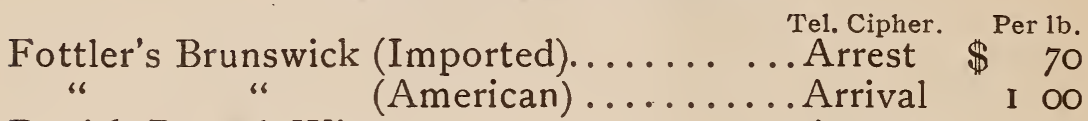

Danish Round Winter............................................. 250

Stone Mason Marblehead .............................. I

Marblehead Mammoth...............Arson 85

Sure Head (American)........................... I 00

Succession (Imported) .................................. 85

" (American)................................... I 25

Green Glazed (American).............................. I 0

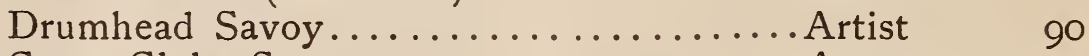

Green Globe Savoy.......................................... I OO

Red Dutch (Blood Red)...............Artesian I 25

\section{CARROT.}

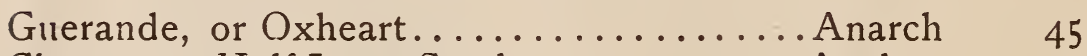

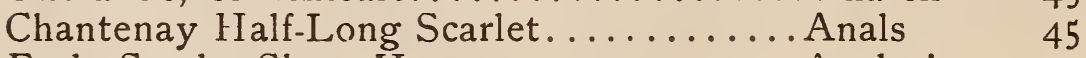

Early Scarlet Short Horn...............Analysis 40

Half-Long Red, Stump-rooted Nantes.....Anatomy 40

" " Iuc (fine, thick sort).........Ancestor 35

“ " Danver's Orange............Ancient 35

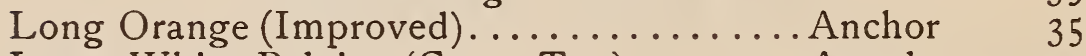

Large White Belgian (Green Top).........Angel 25

"Orange " " $" . . .$. ...Angelo 30

\section{CELERY.}

New Paris Golden (Self-blanching) ........Asbetos 250

New White Plume " " ........Ascension I 25

Boston Market (American) .............Ashes 80

Half Dwarf White........................................ I oo

Golden Heart, or Golden Dwarf...........Aside I oo

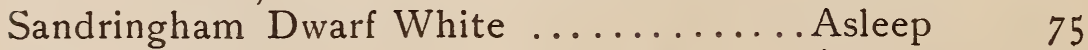

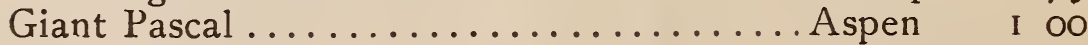

Incomparable Dwarf White ................................. I 0

"Crimson ............Aspirant I oo

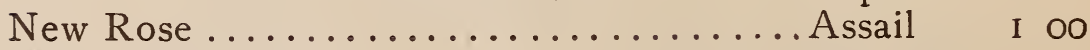

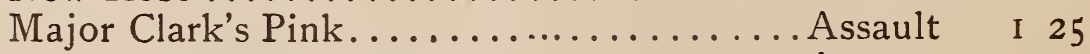

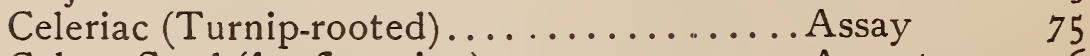

Celery Seed (for flavoring)...................... I6

\section{CHICORY.}

Large Rooted (for Coffee).............Apron 


\section{CAULIFLOWER.}

Early Cipher. Animal

Erfurt Earliest Dwarf ...........Anew

Early London or Dutch ........ Animate

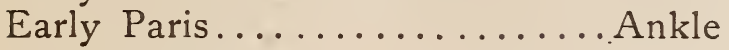

Early Walcheren.............Annex

Half Early Paris, or Nonpareil... Andrew

Lenormand's Short Stem ........Antique

Large Algiers...............Anxious

Veitch's Autumn Giant........

$\begin{array}{rrrr}\text { Per oz. } & \text { Per } 1 \mathrm{~b} . \\ \text { \$I } 75 & 24 & \text { OO } \\ \text { I } 75 & 20 & 00 \\ 25 & 2 & 50 \\ 50 & 5 & 00 \\ 25 & 2 & 50 \\ 40 & 4 & 00 \\ 50 & 5 & 00 \\ 50 & 5 & 00 \\ 35 & 3 & 50\end{array}$

\section{COLLARDS.}

True Georgia, or Creole..............Aurora

\section{CORN.}

CAUTION.-Sweet Corn in bulk, even after being thoroughly cured, will often gather moisture and spoil, particularly if it has been exposed to a low temperature. To prevent this it should be taken out of the bag as soon as received and spread in a dry place. Our Quotations are per measured bushe1, shelled. A11 Eastern Grown.

CROSMAN BROTHERB.

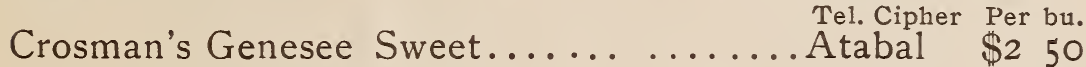

Extra Early Adams................................. 200

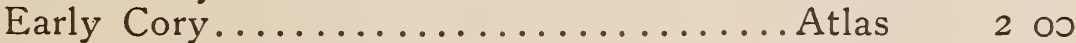

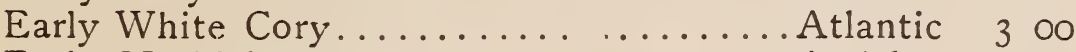

Early Marblehead..................Attick 2 o०

Early Minnesota.................................... 2 ००

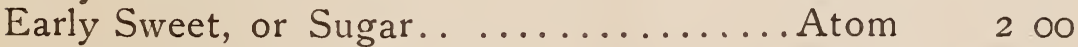

Country Gentleman, "New"..........Atomize 225

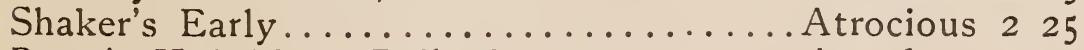

Perry's Hybrid, or Ballard............Attack 200

Crosby's Early...................Attain 225

Stowell's Evergreen, (Eastern Grown)......Attention 200

Moore's Early Concord...............Attire 200

Potter's Excelsior........................................... 225

Clark's None Such (New Variety).........Attitle 225

Egyptian, or Washington Market.........Attorney 200

Hickox Improved. (Fine for Canning)......Attract 200

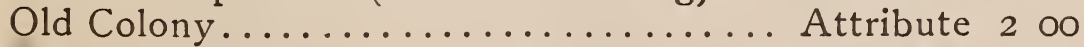

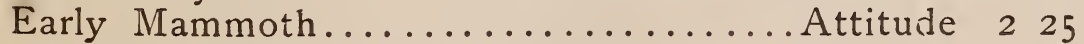

Large Late Mammoth...............Attone 200

Black Mexican...................Attest 225

Parching Pearl (ears).......36 lbs. to bu. Attempt I 50

" Rice "......" " " Attach I 50 
IO CROSMAN BROTHERS' IVHOLESALE PRICE'LIST.

\section{FODDER CORNS.}

Southern Sheep Tooth Fodder, per bag,

( $2 \mathrm{r} / 2 \mathrm{bu}$.), $\$ 2.25$, no charge for bags.... Auction 90

Red Cob Ensilage, per sack (2 I/2 bu.) $\$ 225$,

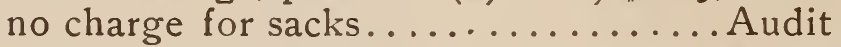

90

Sweet Corn (to sow for fodder)...........A Auger I 25

CORN SALAD, or FETTICUS.

Large Seeded (Large Leaved)........................... Cipher.

Per $1 \mathrm{~b}$.

Improved Green Cabbaging ............Autopsy

35

45

CRESS.

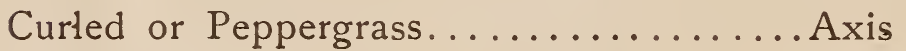

20

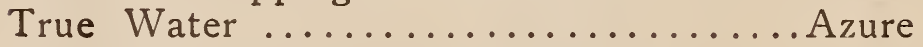

225

\section{CUCUMBER.}

Early Tel. Cipher

Early Russian.............Avail

Early Green Cluster ..........Avarice

Early Short Green............Avast

Early Frame..............Avenge

Early White Spirse............Avenue

Green Prolific..............Average

Boston Pickling.............Avoid

Chicago Pickling..............Avow

Nichol's New Medium Green.... Avowal

Long Green................Averil

West India Gherkin, or Burr ...A Avulsion roo $1 \mathrm{bs}$. Per $1 \mathrm{~b}$.

$\$ 2500$

2500

2500

$25 \mathrm{co}$

2500

2500

2500

2500

2500

3000
30

30

30

30

30

30

30

30

30

35

75

ENGLISH FRAME VARIETIES.

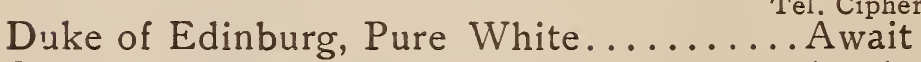

Tel. Cipher. 100 seeds.

Goliath, 20 to 25 inches long, Prolific.......Awake 200

Rolison's Telegraph, Most Prolific........Aware

Model

Award

Giant of Arnstadt, 20 to 25 in. long, white. Away

125

I 50

Glory of Erfurt, Snow White, Extra.......Awful

I $\mathrm{OO}$

100

\section{DANDELION.}

Improved (a fine Spring Salad)........................ Pachelor I 50 Improved Large Leaved, Early.......... Backward 350 


\section{EGG PLANT.}

Early Long Purpler. Tel. Cipher. Per lb.

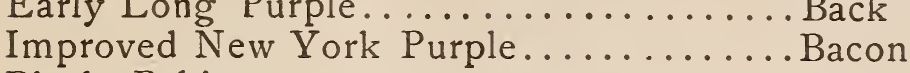

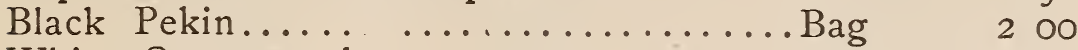
White Ornamental................Bail

ENDIVE.

Moss Curled......................Balance

White Curled...................................

Green Curled, Winter...............Baldness

Broad-leaved Batavian.............. Baleful

White or Lettuce-leaved ....Bale

I 25

90

yo

80

80

GARLIC.

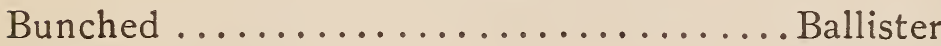

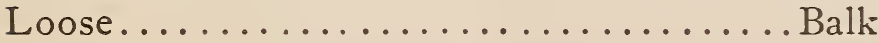

KALE, or BORECOLE.

Dwarf Green Curled Scotch............Ballast

“ Purple Curled................Ballinda

Tall Green Moss Curled...............Baloon

\section{KOHL-RABI.}

Early White Vienna, Short, Small Leaves.. Bamboo I 25 Early Purple " " " " . Banana I 50

Large Late Green, or White............Band 75

\section{LEEK.}

Large Musselburg (Fine American)........Banisher I o०

" Carentan Winter ...............Banknote I o

London Flag (Imported)...............Banquet 75

LETTUCE.

Crosman's New Improved, black seed ..... Baptism 55

Early White Cabbage, white " ......Barr 50

" Curled Simpson white " ...... Barber 45

" " "

“ Prize Head

Big Boston,

Boston Curled, Market,

Deacon, Cabbage,

Denver Market,

Eureka,

Hanson, black

white

white

black “

white "

black

black

brown "

white
. Barefoot . Bartlet

Barton

. Bargain

Barkless

. Barnabee

. Barnacle

. Barometer

Baron 


\section{LETTUCE--Continued.}

Large India,

Buttercup,

Grand Rapids,

Henderson's New York,

Early Tennis Ball,

American Gathering,

Ali the Year Round,

Salamander Head,

Paris White Cos,

Giant White Cos,
Tel. Cipher. Per lb. white seed....... Barnum \$ 50 white " ......Barroness 50 white " ......Barracan 50 white " ......Barricade 50 white " ......Bath 50 white " ..... Battery 50 black " ......Battle 50 black " ......Batten 50 white " ......Bay 60 white " .....Bazar 60

\section{MARTYNIA.}

Martynia Proboscidea, for pickles ........ Beach

\section{MUSHROOM SPAWN.}

Best English, in bricks, per Ioo lbs., \$8..... Bewail

\section{MUSK MELON.}

Extra Early Hackensack .... .......... Beader

Early Prolific Nutmeg ................ Beacon

Fine Large Green Nutmeg .............Beadle

Golden Netted Gem ................. Beard

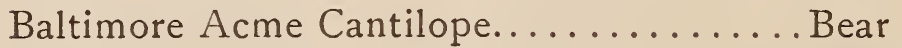

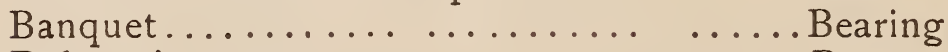

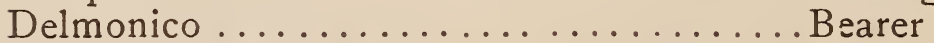

Skillman's Fine Netted ............... Beauty

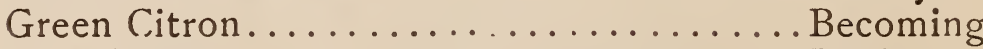

Pine Apple.

Beef

Jenny Lind . . . . . . . . . . . . . . . . . . Before

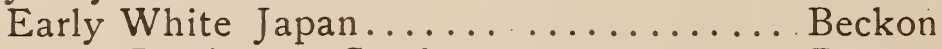

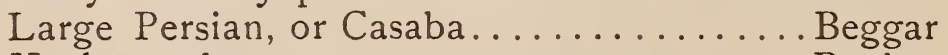

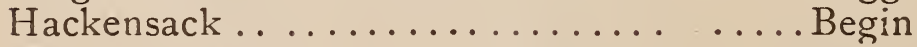

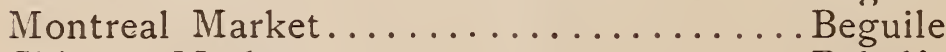

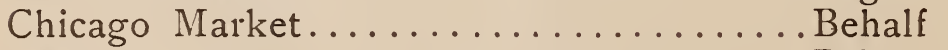

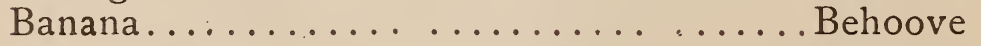

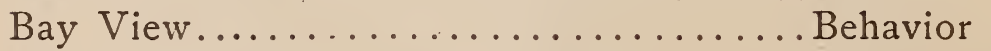

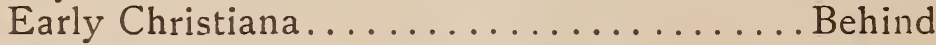

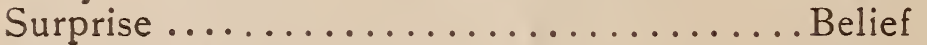




\section{WATER MELON.}

Ruby Gold ................................... \$ $\$ 30$

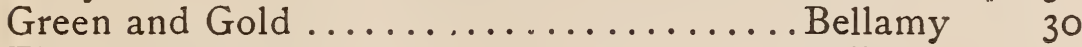

Florida Favorite ........................... 30

Pride of Georgia ...................... Bellows 30

Girardeau New Favorite ................Belock 30

Hungarian Honey....................Belong 25

Seminole ......................... Belfry 25

Mountain Sweet .........................

"Sprout......................nap 25

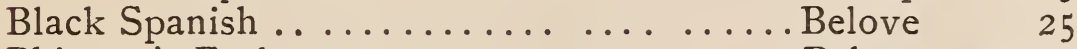

Phinney's Early ..................... 25

Ice Cream, White Seeded or "Peerless"...Belvidere 25

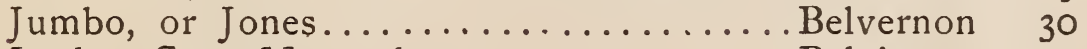

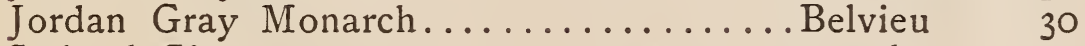

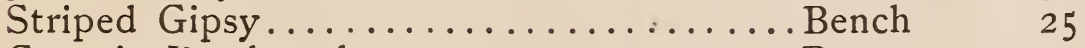

Georgia Rattlesnake.................Benton 25

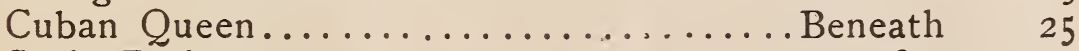

Scaly Bark............................. 30

The "Boss"........................ Berth 25

Mammoth Iron Clad............................ 25

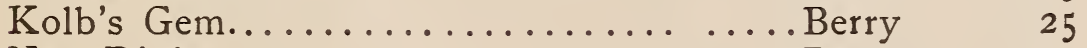

New Dixie ............................ 30

Orange.................................. 40

Volga .................................... 30

Sculptured Seeded Japan ..............Between 40

Citron, for preserving................. Beyond $\quad 25$

MUSTARD.

White, or English........................ I2

Giant Southern Curled............................ 35

NASTURTIUM.

Tall Mixed Colors...................... I oo Dwarf " " Tom Thumb...........Billiards I 0 OKRA.

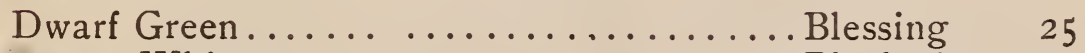

" White .................... Blockade 25

“ New White Velvet Pod...........Blood 25 


\section{ONION.}

\section{AMERICAN VARIETIES.}

These Prices are Subject to Change without Notice.

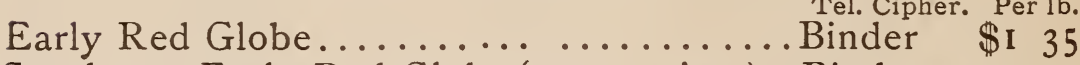

Southport Early Red Globe (new variety)..Bindery I 75

Large Red Wethersfield....................................... 75

Yellow Dutch, or Strasburg............Bird I o

Yellow Danvers....................................... I 0

" " Globe...................................... I 25

" " " " "xtra, ourown growth.Bismarck I 50

White Globe, true................................... 225

White Portugal, or Silver Skin..........Birch 225

El Paso, or Large Mexican.............Bivalve 200

Prize Taker.................................. I 50

IMPÓRTED VARIETIES.

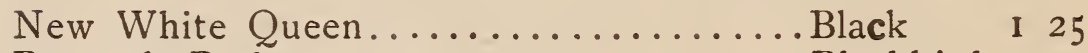

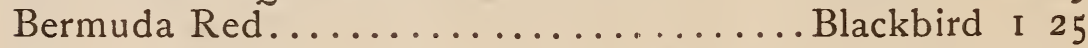

Giant Rocca, of Naples................................. I oo

Early White Flat Tripoli.............................. I 25

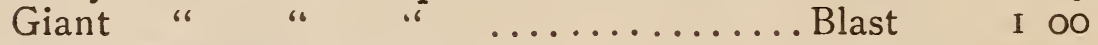

Large Dark Red Bassano.............................. I 25

Mammoth White Garganus, or Silver King.Blazon I 25

ONION SETS.

Prices constantly changing.

Yellow Onion Sets......... Blair

Red " " $\quad \ldots . . . .$. . Blarney $y$ Price on applicat'n.

Top or Button Onions.... .. Blame

Potato Onions, or Multipliers. Blast

\section{PARSLEY.}

Beauty of the Parterre............... Bluster

Extra Double Curled ................. Blossom

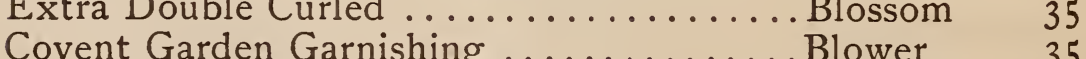

Myatt's Garnishing................................

Fern-learned (extra fine)............... Bloomer $\quad 35$

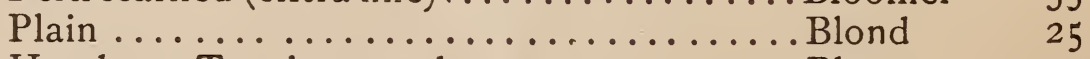

Hamburg Turnip-rooted ............... Bloom 25 


\section{PEAS.}

Those marked (*) are Wrinkled Varieties. All Garden Peas carefully Sifted and Hand-Picked.

EARLY.

Crosman's First and Best................................. \$3 OO

Extra Early "True".......................................... 275

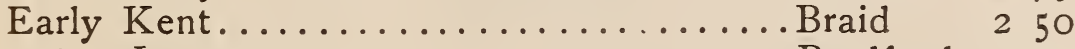

" June................................................. 250

Dan. O'Rourke (Improved)............................ 250

Philadelphia Extra Early................................ 250

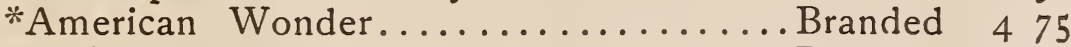

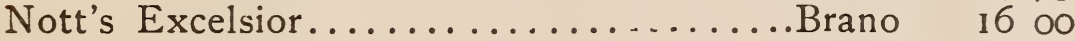

*Extra Early Premium Gem............Brass 400

* McLean's Little Gem.................................... 400

Blue Beauty................................................ 325

McLean's Blue Peter. .............................. 400

Tom Thumb (American).......................... 425

Pride of the Market...................................... 5 oo

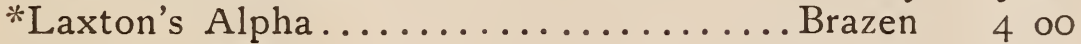

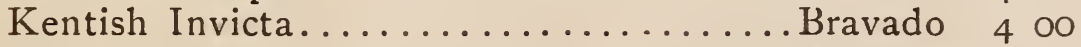

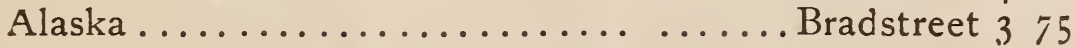

SECOND EARLY.

*Bliss' Abundance....................................... 350

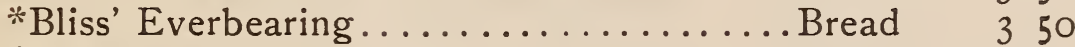

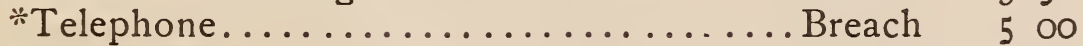

*Stratagem ...................................................... 50

*McLean's Advancer.......................................... 375

*Horsford's Market Garden.............Breathe 4 00

*Shropshire Hero.................................... 525

*Champion of England............................... 275

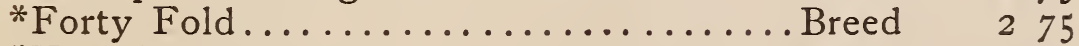

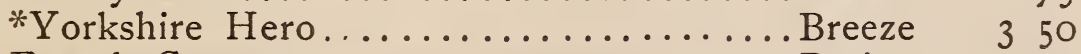

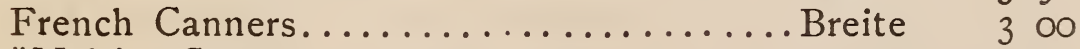

* Melting Sugar.......................................... 700

Dwarf Sugar (Edible Pods)................................ 500

Tall " " "............Breck 5 co

Large Dwarf White Marrowfat..........Brent I 60

Black-Eyed Marrowfat...................... I 60

FOR FIELD CROP-(All thoroughly Screened).

Canada Field, First Quality.......................... I I 5 " " Second Quality........... Brickred I 00

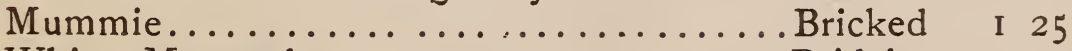

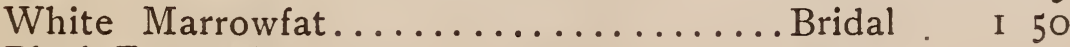

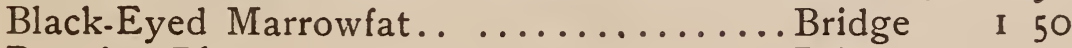

Prussian Blue.............................. I 25 


\section{PARSNIP.}

Te1. Cipher. Per 1b.

Long White Dutch.................. If

Improved Hollow Crown.............Blunder I8

Gurnsey, or Cup.................... I

\section{PEPPER.}

Large Bell, or Bull Nose ...................... I 25

Large Squash, or Tomato Shaped.........Boaster I 25

Sweet Mountain, or Mammoth...........Boatman I 25

Monstrous, or Grossum................. I 75

Golden Dawn........................... I 25

Ruby King ............................. I 50

Red Chili ............................... I 50

Long Red Cayenne................... Bodily I 25

Long Yellow Cayenne.................... I 50

Cardinal................................. I 50

New Celestial............................ I 50

Red Cluster ..................... I 50

Cherry (Small Red)..................... I 50

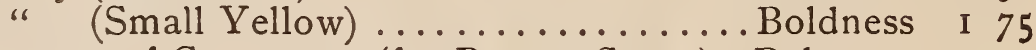

Cayenne of Commerce (for Pepper Sauce)..Bolster 200

\section{POTATOES.}

At prices quoted we make no charge for barrels or cartage. ( $16_{5} \mathrm{lbs}$. in a bbl.)

Tel. Cipher. Per bbl.

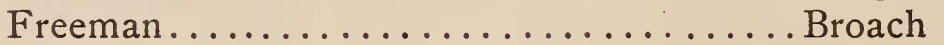

400

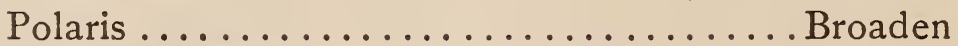

325

Early New Yorker.........................

Early Ohio Junior........................ Bro

Clark's No. I....................... Brow

Early Beauty of Hebron............... Broth

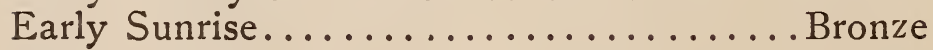

325

400

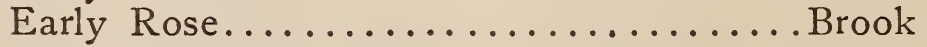

Chicago Market.................. Brown

Early Puritan.................... Bromide

3.00

250

325

325

300

Brownell's Winner.............................

325

Prize Taker..................... Brotop

300

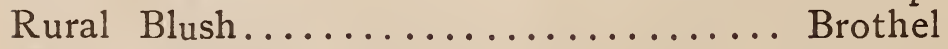

325

Maggie Murphy................... Brother

250

Rural New Yorker No. 2................ Brucite

400

Rochester Rose..................... Brubach

American Wonder...................Bruder

Troy Seedling....

Brucina 


\section{PUMPKIN.}

Cushaw

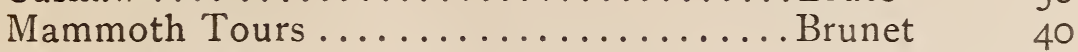

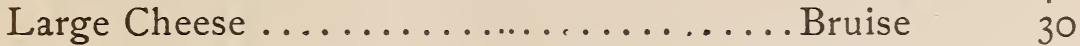

Small Sweet, or Sugar .............................. 30

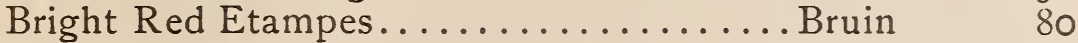

King of the Mammoth................................ 75

Connecticut Field, bushel of 28 lbs., $\$ 2.50$.. Bruso I2

\section{RADISH.}

French Breakfast.......................... 30

Early Deep Scarlet Olive Shaped........Bugle 35

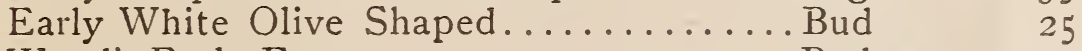

Wood's Early Frame........................................... 25

Rosy Gem White Tipped .......................... 30

Early Scarlet Turnip-Rooted.............Buffet 25

Early White " " ........................... 25

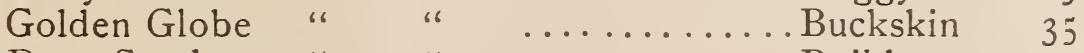

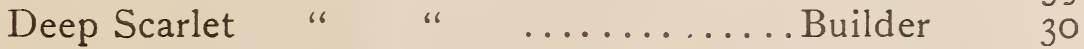

White Tipped Scarlet " ...........Bullet 30

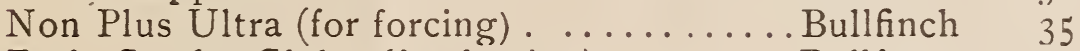

Early Scarlet Globe (for forcing)......... Bullfrog 45

Philadelphia White Box.................................. 35

Long Scarlet Short Top..............Bullock 25

Long White Vienna, or Lady Finger......Bullrush 45

White Strasburg, or Hospital......................... 40

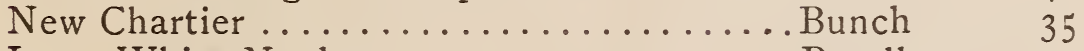

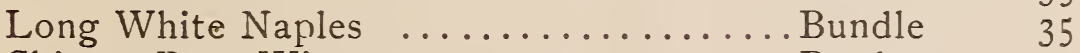

Chinese Rose Winter ...............Burden 40

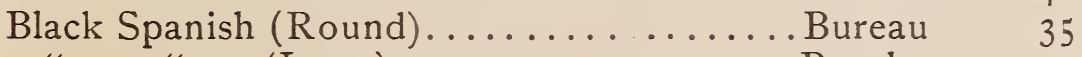

" " (Long) ................Burglar. 35

California Mammoth White Winter .......Butcher 35

RHUBARB.

Linnæus.................................. 60

Victoria ........................................ 50

Roots of Victoria, per I00, $\$ 4.00 \ldots \ldots$..... Buttress

\section{SALSIFY.}

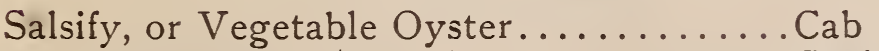

New Mammoth Sandwich Island......... Cabin

\section{SUNFLOWER.}

Mammoth Russian, per $100 \$ 5.00 \ldots . .$. Cage

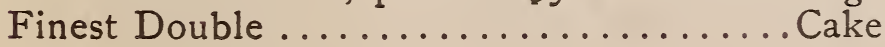




\section{SPINACH.}

Round. Cipher. Per th.

Round, or Summer.............................. Io

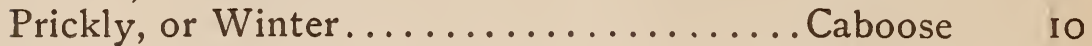

New Thick-Leaved Viroflay ............................. Io

Savoy-Leaved, or Bloomsdale......................... I2

Long Standing............................ I 2

\section{SQUASH.}

SUMMER VARIETIES.

Early Yellow Bush Scallop ............ Calcium

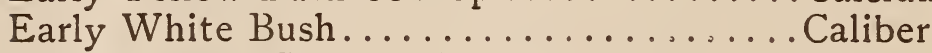

Early Golden Crookneck.. ...........Calm

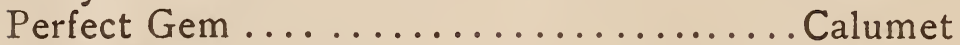

30

30

30

35

FALL AND WINTER VARIETIES.

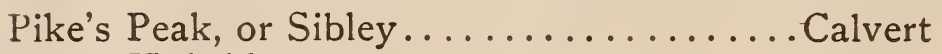

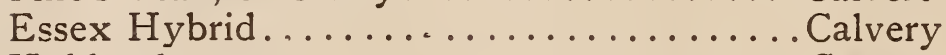

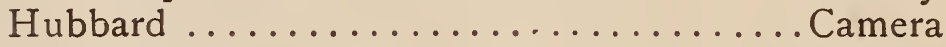

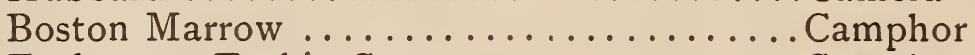

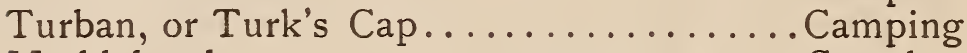

Marblehead...................Camel

Butman

Mammoth Chili.
- Campana

-. Campaign
30

35

40

25

35

30

30

75

\section{TOBACCO.}

Connecticut Seed Leaf. ................. Canal

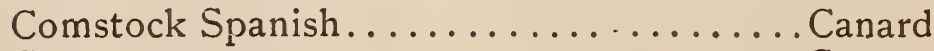

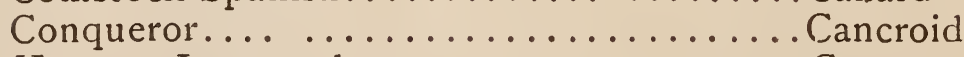

Havana, Imported...........................

Havana, Seed Leaf.............................

Little Dutch.

Candicint 200

Orinoko Sweet, or Little Orinoko .........Candid I 50

Orinoko Yellow .................................. I 50

Sterling .................................

Tuckahoe

. Candock

I 50

White Burley, Superior Strain

. Candroy

I 50

TOMATO.

Dwarf Aristocrat (new) .................................... I 75

Buckeye State (new)......................................... I 75

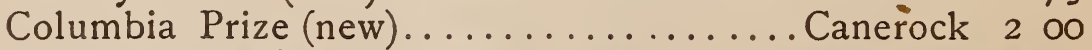

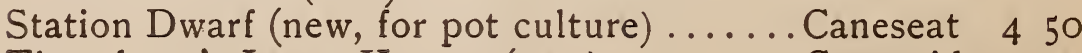

Thornburn's Long Keeper (new)......... Caneroid I 75 


\section{TOMATO-Continued.}

Early Atlantic Prize

Tel. Cipher. Per 1b.

Early Ruby...

Canehole I 50

New Stone

Canhook I 50

Ponderosa

Canicule I 50

Royal Red

Canine Sold out

Crown Jewel.

Canister

Candidate i 50

Volunteer

Candiot

I 50

Ignotum

Candy

Beauty...

Candle

Favorite

Cane

Perfection

Canker

150

Acme.

Cannon

I 25

Paragon

Canue

Mayflower.

Canopy

I 50

Mikado

Canteen

Turner's Hybrid

Cantel

I 25

Scovill's

Cantilate

I 25

Dwarf Champion.

General Grant.

Canton

I 25

Excelsior.

Canvas

I 25

Canada Victor

Capable

I 25

Conqueror

Caper

I 25

Trophy

Queen "Red".

Captive

I 50

Hubbard's Curled Leaf

Capon

I 50

I 50

Fejee Island.

Caprice

I 50

I 25

I 25

I 00

I 25

I 50

Captain

I 25

Caption

I 25

Golden Queen, or Sunrise

Carbine

Golden Trophy

Green Gage. .

Caramel

I 50

I 50

Peach

Carapax

I 50

Lorillard

Card

I 50

Table Queen

Cardboard

I 25

Optimus

Carding

25

Pear-Shaped Yellow.

Carey

I 50

Pear-Shaped Red

Career

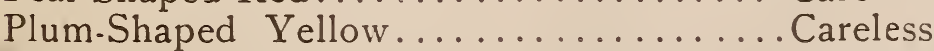

I 25

Plum Shaped Red

Caress

Yellow Cherry...

Cargo

I 50

Red Cherry

Carman

Red Apple

Carlo

Red Currant

Cartoon

I 50

Strawberry, or Ground Cherry

Carnival

I 50

I 50

I 50

I 50

I 50

200

I 75 


\section{TURNIP.}

NOTE.-Ten pounds or more are invoiced at roo-pound rates.

Te1. Cipher. roo lbs.

Per $1 b$.

Extra Early Milan, Strap-Leaf..Cascade Sold out. Early White Flat Dutch .......Cashier 2000 Stone ........... Caster

2000

Early Six Weeks or Snowball... Casual

2500

Purple Top, Strap-Leaf ....... Catacombs 2500

White " "...... Cataract 2000

Purple Top, White Globe .. ... Cater

2000

Early White Egg........... Cateress 2500

Montmagny

Cathedral

Purple Top Scotch, or AberdeenCattle

3000

Large White Globe, Pomeranian . Caustic

2000

2000

Norfolk

Cavalcade

2000

Cow Horn, Long White........ Cavalier

2500

2500

Robertson's Golden Ball ....... Cavalry

Seven Top (for Turnip Greens). . Cavern

2000

\section{RUTA BAGAS, or SWEDES.}

Skirving's Improved Purple Top.Cedar

Carter's Imperial Purple Top... Ceiling

1600

American Purple Top Ruta Baga Celiac

Laing's Improved Purple Top... Cellar

Shamrock Swede Purple Top... Cement

I6 00

White Sweet, or White Russian. Censor

2000

Sweet German.......................

2000

25

2000

25

2200

2200

25

\section{MISCELLANEOUS SEEDS.}

Broom Corn, Evergreen.......Despair 500

08

Flax Seed............... Desperate 350

08

Honey Locust..............Despisal 2000

Lathyrus Sylvestris..........

Osage Orange............. Despond 3500

Spring Vetches.............Despot

Sugar Cane, Early Amber....... Destiny

" Early Orange...... Destroy

450

500

25

250

40

Teosinte

500

08

08

Wild Rice (Zizania Aquatica).... Desto

Rape, best Essex............ Dessere

I 500 


\section{$\rightarrow$ HERBS愔}

\section{SWEET AND POT HERBS.}

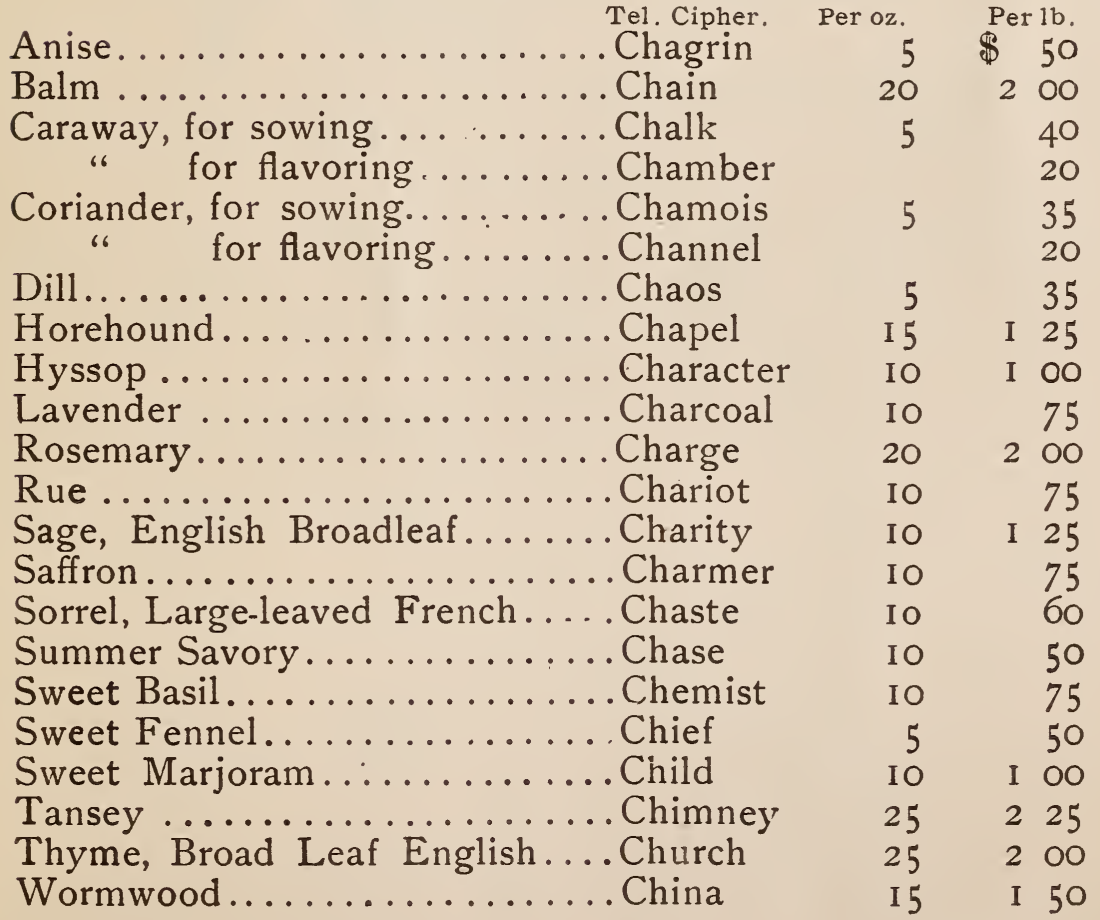




\section{GRASSES AND CLOVER.}

Red Top-I4 lbs.-(Agrostis Vul-

garis $) \ldots \ldots \ldots \ldots \ldots \ldots$ Daily

Red Top-I4 lbs._Extra Clean... Dace

Red Top, "Fancy," Clean....... Daint

Kentucky Blue, Clean.......... Daker

Kentucky Blue, extra clean, I4

lbs.-(Poc Pratensis) ........ Dancer

Kentucky Blue, Fancy, clean...... Dandy

Orchard Grass-I4 lbs.-(Dactylis

Glomerata $\ldots . . . \ldots \ldots$........ Daunt

Crested Dog's Tail (Cynosurus Cris.

$$
\text { tatus) ................... Debar }
$$

Fescue, Fine Leaved (Festuca Tin-

uifolia)................ Declare

Fescue, Hard-Leaved (Festuca Du-

Per bu. Per $1 \mathrm{~b}$.

\$I 00

IO

I 40

15

20

75

I $25 \quad$ IO

I $60 \quad 15$

$250 \quad 20$

riscula ................. Defence

Fescue, Sheep's (Festuca Ovina)... Depot

Fescue, Meadow (Festuia Pratensis)Defer

Italian Rye Grass (Lulium Italicum). Deponent

Pacey's Perennial Rye-24 lbs.-

(Lolium Perenne)........... Depress

Yellow Oat Grass-8 lbs.-(Avena

Flavescens $). . . \ldots \ldots \ldots$...... Delay

Bermuda Grass................ Dell

Sweet Vernal (Anthoxanthum Odo-

ratum) Perennial...........Delta

Hungarian Grass-48 lbs.-(Pan-

icum Germanicum).......... Deputy

German or Golden Millet........ Depth

Fine Mixed Lawn Grass-I 5 lbs.. Derive

Lawn Grass, Crosman's extra fine

Park Mixture-I 5 lbs....... Descend

Clover, White Dutch, per Ioo lbs.,

$\$ 30.00 \ldots \ldots \ldots \ldots \ldots$ Desert

Clover, Alsike or Swedish, per Ioo

lbs., $\$ 15.00 \ldots \ldots \ldots \ldots \ldots$ Design

Clover, Alfalfa, or Lucerne, per 100

lbs., \$1 $\$ 2.00$............. Desolate

I $75^{\circ} \quad 12$

40

30

I 8

15

15

I $50 \quad 12$

$480 \quad 65$

Expected.

I 25

225

275

Clover, Crimson Trifoliam, incarna-

tum, per IOo lbs., $\$ 7.00 \ldots$.... Desolve 


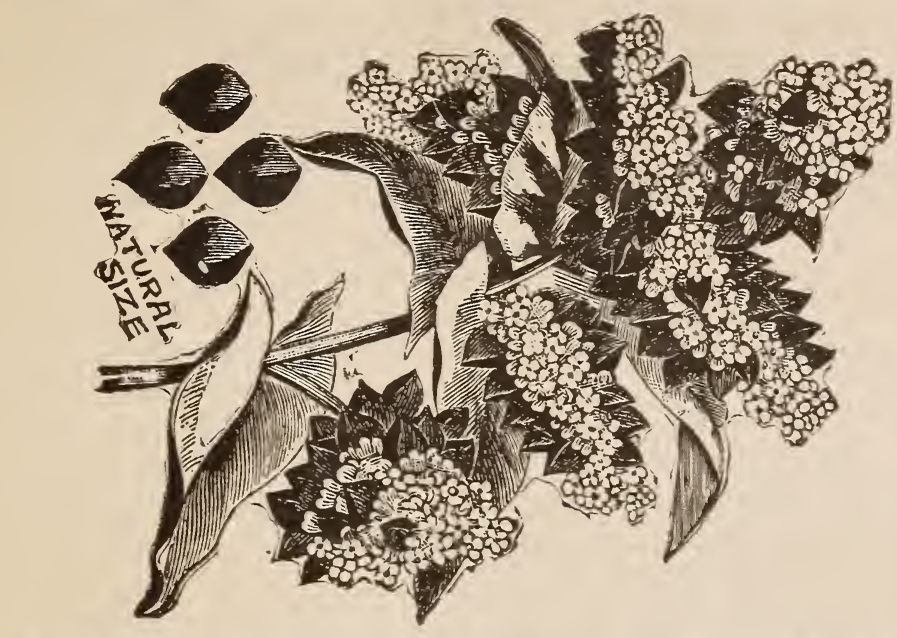

STANDARD FIELD GRAINS.

\section{BUCKWHEAT.}

Tel. Cipher. Per roo lbs. Per bu.

New Japanese................ Each \$I 65 \$ 80

Silver Hull.................. Eagle 2 ०० I ००

\section{CORN, Tele. Cipher.}

Angel of Midnight, Yellow Flint..........Eager I IO

Extra Early Huron, Yellow Dent..........Ear I Io

King of the Earlies, Yellow Dent.........Earache I o

Golden Beauty, Yellow Dent.........Earl I IO

Longfellow, Yellow Flint.........Earlap I IO

Leaming, Improved, Yellow Dent.........Earth I Io

Pride of the North, Yellow Dent.........Earthly I o

Sanford, White Flint.........Ease I Io

Southern Sheep Tooth, for Fodder.........Auction 90

Red Cob Ensilage, " $\quad$.........Audit 90

Sweet Corn,

" .......Auger

I 25

OATS $-32 \mathrm{lbs}$.

The Great White Main...............Eatable 70

Mammoth Cluster...................Eating 80

Early White Swede................... Eaves 65

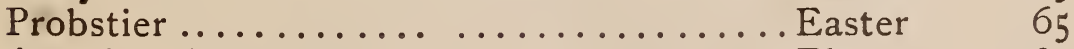

American Banner...................... Ebony 80

RYE.

Choice Spring ( 56 lbs.).............. Eclat I 50

"Winter " ..............Echo I 25 


\section{GENERAL LIST \\ -OF- \\ $\rightarrow$ FLOWER SEEDS, 恼}

BY ILEIGHT.

Of Varieties priced at Fifty Cents per Ounce or under, not less than half ounce will be furnished at ounce rates. Quarter pound at pound rates.

Abronia Umbellata................. \$I $75 \$ \$ 20$

" Arenaria.................... 40

Aconitum (Monk's Hood)................. ... 75

Accroclinium, Mixed, "Everlasting Flowers" I 25 I 5

Adluminia Cirrhosa...................

Adonis Aestivalis, Red............... $60 \quad 10$

Ageratum Mexicanum, Blue........... I $25 . \ldots$ I 5

" " " $\quad$ Nanum, Blue.... $350 \quad 35$

" " Nanum "6 "

" " Swanly, Blue......... $\quad 75$

" Lasseauxii.................. 50

Agrostemma, Mixed Varieties............. I

Alonsoa, Mixed Varieties.................. 20

Alyssum, Sweet................. I 25 I 5

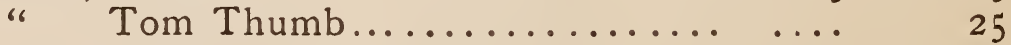

" Saxatile Compactum............. 25

Amaranthus Tricolor.................. I 5

" Caudatus.................. IO

" Cruentus.................. 10

" Salicifolius................. 40

" Mixed Varieties.......... $40 \quad$ IO

Antirrhinum Majus, Mixed, Snap Dragon.. $200 \quad 20$

"Tom Thumb varieties, Mixed. ... 40

Asperulia Azurea Setosa............ $40 \quad$ IO

Aster, $\mathrm{Ne} w$ Rose, mixed colors............. 300

"Truffaut's Pæony-flowered Perfection .... 250

"Victoria, mixed colors.............. 500

" Dwarf Bouquet, I 5 mixed colors.... .... 300

" "Crysanthemum fl'd,mixed colors

"Imbique Pompon, " " "

" Cocardeau or Crown,

" Pæony Flowered Globe, " " "

"Washington,

$66 \quad 66$

300

300

300

300 
Per 1b.

Balsam, Camelia Flowered, mixed colors... \$.... Rose Flowered,

Fine double mixed.

Bartonia, Aurea.

Brachycome Iberidifolia, Swan River Daisy

Briza Maxima, "Quaking Grass"........

Caccalia Coccinea.

Calendula, Meteor.

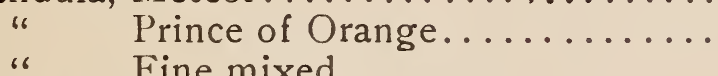

Calliopsis, finest mixed colors.

Campanula Medium

(Canterbury Bell)

Double Mixed. .

(Canterbury Bell)

Single Mixed... I 50

6

66

"6

Calycanthema, Mixed

Colors . ........

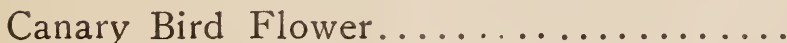

Candytuft, White

300

Lilac.

50

Purple.

I 25

I 25

-Extra Dark Crimson......... I 25

Mixed Colors................

Canna, Mixed varieties.

60

I 00

“ Dark-leaved sorts..................... 2 50

" Madame Crozy.

Carnation, extra fine, double mixed.......

Good mixed.

66

Grenadin, brilliant scarlet

Margaret, mixed colors .........

Catchfly, mixed colors................

Celosia, dwarf mixed, "Cockscomb".....

" Japonica, "Japan Cockscomb"...

Centaureả, Emperor William............

" Cyanus, Bachelor Button, minor

Centranthus, mixed colors..............

Clarkia Elegans, double var. mixed... single

Cobæa Scandens, purple. .

Columbine Aquilegia, double mixed colors.

Convolvulus Minor, mixed.
Per oz.

$\$ 60$

75

30

I 5

50

IO

20

IO

IO

IO

IO

60

I 5

I $\mathrm{OO}$

30

IO

I 5

I 5

I 5

IO

10

25

40

60

250

I 50

300

I 50

IO

I 00

60

I 5

IO

IO

15

I 5

40

I 5

IO 
Cypress.

Cypress Vine, Scarlet, White, Rose, each.. \$I 25 mixed colors........... I 25

Dianthus Chinensis, mixed varieties...... 200 Per oz. “ Imperialis (Double Imperial Pink) “ Heddewigii Diadematus.

250 66

Heddewigii and Laciniatus, mixed colors........ 500

Digitalis, mixed (Foxglove)..

Double Daisy, mixed colors, from select flow's " " " " very fine quality.

Datura Wrightii.... " white fl. pl......

Dolichos Lablab (Hyacinth Bean)........ Eschscholtzia, mixed colors........... I o Euphorbia

$$
\text { Heterophylla. }
$$

Fraxinella, Red and White mixed........

Gaillardia Grandiflora, splendid mixed var. . Gilia, mixed varieties.............. Godetia, mixed colors.

Gomphrena Globosa, mixed (Globe Ama-

$$
\text { ranth) }
$$

Gomphrena Globosa, Orange, Aurea Superba Gourd, Ornamental, mixed var., small sorts.

$$
\text { " " " large sorts. }
$$

Gypsophila Paniculata................ Helianthus Globosus, finest double Sunflower Mammoth Russian........... Helichrysum, mixed, "Everlasting Flower" Helipterum Sandfordii.............. Hollyhock, finest double, mixed......... Ice Plant. Ipomopsis, mixed colors ................ Ipomœa Coccinea (Star Ipomoea)........ I oo

...

...

"Grandiflora Hybrida Alba (Moon Flower).

Lobelia, "Crystal Palace" Speciosa.......

Loasa Aurantiaca...................

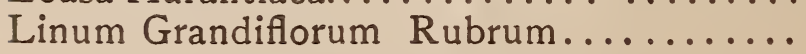


Lupinus, mixed colors.

Per $1 b$. 40

Lychnis Chalcedonica, mixed colors...........

Marigold, French, Dwarf, mixed colors.... I 50

"6 "6 Tall, "6 $\quad$ "...

" African, mixed colors......... I 50

" Tagetes Signata Pumila...... 250

Martynia Proboscidea.

Marvel of Peru,mixed colors, "Four o'clock"

Maurandya, mixed colors.............

Mignonette, sweet, large-flowered.

Grandiflora Ameliorata........

Parson's New White.

Machet.

Golden Queen.

Crimson

Mimulus, Tig

" Moschatus Compactus.

Mesembryanthemum Crystallinum(Ice Pl'nt)

Tricolor (Dew Plant).

Moon Flower, Cross-bred or Hybrid.

Morning Glory mixed, "Convolvulus Major"

Myosotis Pălustris, "Forget.Me-Not "....

Nemophila, mixed colors.

Nasturtium, Dwarf, mixed colors ...........

Tall, mixed colors............

Nicotiana Affinis.

$$
\text { " Tall, mixed colors }
$$

Nigella, double, Damascena, fl. pl ..........

Enothera Lamarckiana Grandiflora........

I 50

35

....

50

60

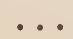

$\ldots$

200

200

...

…

I 50

....

700

30

...

50

I 00

I $\mathrm{OO}$

....

50

Pansy, very fine, German mixed.........

600

Finest quality, mixed.......... I2 00

very large flowering, mixed........ I 500

Bugnot's, Superb Blotched........ ....

Benary's Non Plus Ultra...........

Cassier.

Odier, or Blotched.

Trimardeau, or Giant............

Azure Blue.

Atropurpurea Alba Marginata......

Gold Margined.

...

...

$\ldots$

$\cdots$

$\cdots$

. . .

King of the Blacks...............

Lord Beaconsfield

Emperor William

Princess Bismarck.
Per oz.

IO

25

20

20

20

25

20

IO

I 25

IO

IO

30

50

20

20

300

250

20

30

70

5

400

IO

IO

IO

25

IO

20

60

I 25

I 50

800

400

600

400

225

75

85

75

85

85

85

75 
Per $1 \mathrm{~b}$.

Pansy, Quadricolor.................\$\$

Silver Edge.

$\cdots$

$\$$

Per oz.

“ Snow Flake, or Snow Queen.......

“ Striata Perfecta, extra fine strain...

$\cdots$

Purplish-violet, white-bordered.....

"Wallflower color.................

$\cdots$

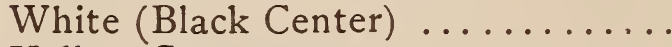

Yellow Gem.

“

Yellow (Black Center)

Pentstemon, mixed varieties.

Petunia, Striped and Blotched

Finest Hybrids, mixed............

Fine Mixed Hybrids.

" Countess of Ellesmere.

40

Phlox Drummondii, mixed colors....... \& 00

$$
\text { “ }
$$

“

66

"،

Grandiflora mixed....

600

40

60

75

66

66

Bright scarlet

75

Perilla Nankinensis Pure white.

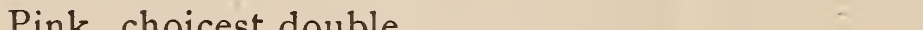

Poppy, The Shirley............... 4 o

Umbrosum

350

" Rhœas fl. pl. Dwarf French....... I

" Carnation, double, mixed colors....

"Pæony-flow'd, double, mixed colors

" Orientale...

Portulaca, double, extra fine, mixed

" Single, mixed.

Pyrethrum (Double Feverfew)..........

250

" (Golden Feather)

Ricinus Sanguineus (Castor Oil Bean)......

“ Duchess of Edinburgh...........

" Borboniensis Arboreus..........

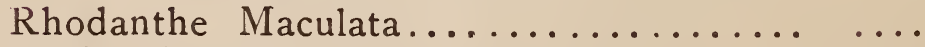

Rocket Sweet, mixed colors............ 50

Salpiglossis, mixed colors........... 400

60

Salvia, mixed colors...................

Scabiosa Majus, Tall, mixed

“ Dwarf varieties mixed......... I 25

Sanvitalia Procumbens fl. pl................

Schizanthus, mixed colors.

I $\mathrm{OO}$ 
Per $1 b$. Sensitive Plant..................\$ 3 \%० Stock, German Dwarf, mixed...............

$$
\text { " " } \quad \text { Large-flowering, mixed.... }
$$

Sweet Peas, Apple Blossom............ 50

Per oz.

$\$ 30$

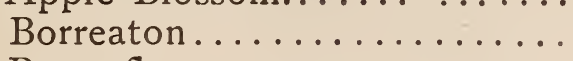

Butterfly

50

Countess of Radnor..........

45

Grand, or Imperial Blue.....

60

Invincible Scarlet.

45

75

225

3 no

$50 \quad 10$

Indigo King ("Purple Prince")

40

Lottie Eckford.

45

Miss Blanche Ferry.........

Mrs. Sankey, white..........

Orange Prince.............

Painted Lady................

Primrose.

Princess Beatrice

45

45

60

75

Princess of Wales

Queen of England..........

Queen of the Isles...........

Senator..................

Splendor.

50

45

50

50

50

Violet Queen..............

45

White ...

50

Mixed Colors...............

40

Eckford's Hybrid, finest mixed

35

Sun Flower, Double Globosus Fistulosus. .

Mammoth Russian.........

IO

Swan River Daisy

10

Sweet William, Double, mixed...........

$$
\text { Single }
$$

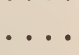

Thunbergia, mixed

Tropæo. um, Tall, mixed colors......... I

Tall, King Theodore.............. 
Verbena Hybrida, fine mixed.........\$ 500

" " very fine quality...... 800

" " " $"$ extra,from named vars.. I 200

" " " Auriculæflora.............

" " $"$ Auriculæflora Cœrulea.. ....

" " " Scarlet Colors........ ...

" " "

" " " Finest Striped........ ...

.6

Mammoth

Waitzia Aurea.

Wallflower, Double, mixed colors............

Whitlavia Grandiflora.............. I o

Zinnia, Double Dwarf, mixed.......... 250

“6

“6

Striped ...

Xeranthemum, Double, mixed colors..........

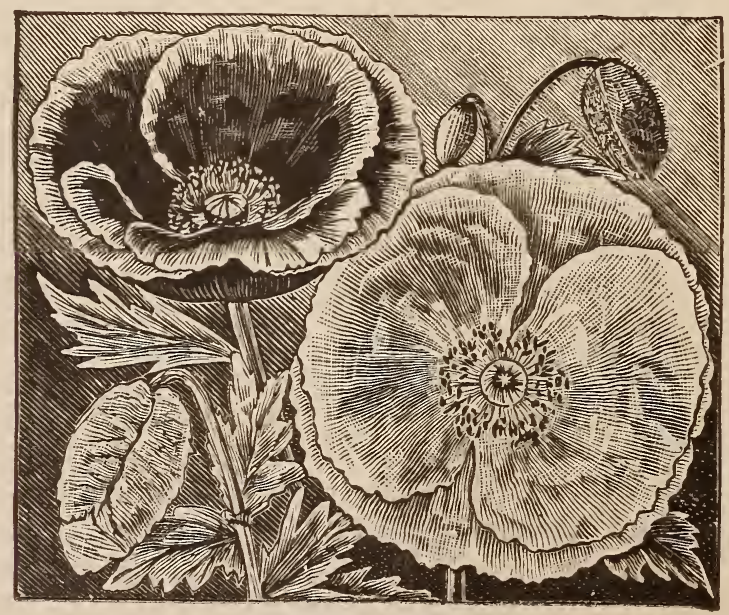

SHIRLEY POPPY. 


\section{Prices of Vegetable Seeds PUT UP IN CABINETS.}

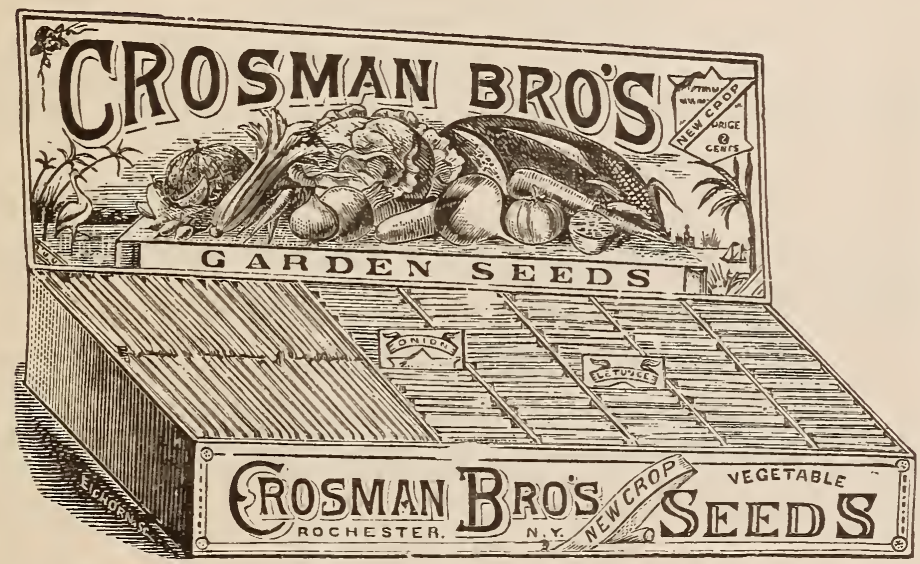

For the convenience of those who may not be familiar with the kinds most needed in their trade, would say we have them put up in neat, flat cabinets, stained pine, of 200,250 and 300 papers each, in such varieties as our long experience has taught us is most needed by the trade generally. We will furnish any number of papers of each kind that may be ordered, or in cabinets as above men. tioned. A list in the form of an order sheet of the varieties, we put up in the above-mentioned style, will be forwarded on application.

Cabinets of 200 papers, valued at $\$ 10.00$, for ....\$4 00

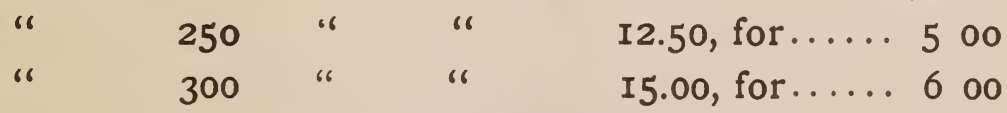

Assorted, per I00 papers, ordered separately..... 250 Corn, Peas and Beans, per Ioo papers (IOc. pps). 350 SPECIAL PRICES FOR LARGE QUANTITIES. 


\section{VEGETABLE PLANTS. \\ If by Mail, add 25 Cents to the Price per 100.}

\section{CABBAGE PLANTS.}

(Transplanted Plants ready April 25th.)

Early Summer, Early Wakefield, Succession, Early Etampes or Express, All Seasons, Winningstadt and Early Flat Dutch; per 100, 75c.; 1,000, $\$ 6.00$.

\section{CAULIFLOWER PLANTS.}

(Transplanted Plants ready April 25th.)

Lenormand's Short Stemmed, Early Erfurt, Early Paris, Early London, and Algiers; per 100, 75c.; $1,000, \$ 6.00$.

Henderson's Early Snowball, per 100, $\$ 1.00 ; 1,000, \$ 8.00$.

\section{CELERY PLANTS.}

(Transplanted Plants ready May 15th.)

Golden Heart, Boston Market, Sandringham, White Plume, Carter's Crimson, Major Clark's Pink, Paris Golden Self Blanching, Giant Pascal and New Rose; per 100, 75c.; $1,000, \$ 6.00$.

\section{PEPPER PLANTS,}

(Transplanted Plants ready May 15th.)

Sweet Mountain, Tomato-Shaped, Monstrous or Grossum, Cayenne, Yellow Cherry, Red Cherry, Large Bell or Bull Nose, Ruby King, Golden Dawn, New Celestial, Red Ćluster and Cardinal ; per 100, \$1.00: 1,000, $\$ 7.50$.

\section{EGG PLANTS,}

(Transplanted Plants ready June 1st.)

Improved New York Purple, Early Long Purple and Black Pekin; per $100, \$ 5.00$.

\section{TOMATO PLANTS.}

(Transplanted Plants ready May 1st.)

Columbian Prize, Mascot, Royal Red, New Stone, Volunteer, Early Ruby, Crown Jewel, Mikado, Acme, Favorite, Paragon, Perfection, Ignotum, General Grant, Trophy, Mayflower, Beauty, Dwarf Champion and many of the New and Fancy Varieties; per 100, $\$ 2.00 ; 1,000, \$ 10.00$.

\section{LATE CABBAGE PLANTS.}

\section{(Ready June 1st.)}

Premium Flat Dutch, Fottler's Brunswick, Drumhead, Stonemason, Marblehead, Sure Head, Savoy and Red Dutch; per 100, 25c.; 1,000, \$2.00.

\section{LATE CELERY PLANTS.}

(Rerdy June 15th.)

All the popular sorts ready in June for planting for winter use; per;100, 50c.; $1,000, \$ 4.00$. 


\section{MISCELLANEOUS.}

\section{Spring Bulbs and Roots.}

Calla, Ethopica (dry roots)............per 100, \$1 500 Caladium, Esculentum, about 2 to 3 in. diam. " " about 3 to 4 in. diam. " " 4 in. and upwards.. Canna, mixed sorts (dry roots)........... Dahlias, Ioo beautiful named varieties..... Gladiolus, choice flowering roots......... Maderia vine, the popular climber......... Tritoma Uvaria (fine).

Tuberose, Pearl, Double White, first size...

\section{ASPARAGUS ROOTS.}

Conover's Colossal, two year old roots..... per I, $000 \$ 400$ " " one " " ...." " 300 Palmetto, new variety, two year old roots. " " " 450

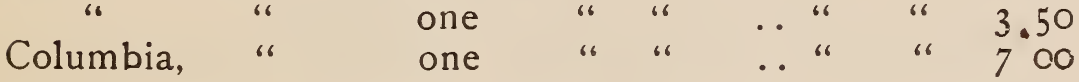

\section{SMALL SERD BAGS MADE OP FIRSTRTLASS PAPRR.} (SAMPLES BY MAIL ON APPLICATION.)

Size-Flower Seed. ............. \$ 25 per I,000 No. I................... I 25

" $2 \ldots \ldots \ldots \ldots \ldots \ldots \ldots \ldots$ I 50 "

" $3 \ldots \ldots \ldots \ldots \ldots \ldots \ldots \ldots \ldots$ I 60 "

" 4 .................... 200 "

$\frac{1}{2}$ pint..................... $225 \%$

$\frac{2}{3}$ pint........................ 275

I pint..................... 225

I pound $\ldots \ldots \ldots \ldots \ldots \ldots \ldots \ldots \ldots \ldots \ldots \ldots$

I quart.................... 325

Printing addresses on any of these, from $\$ 1.00$ to $\$ 2.00$ per 1,000 . 


\section{SEED DRILLS, CULTIVATORS, ETC.}

For Illustrations and Descriptions, see following pages.

BOXED AND DELIVERED ON BOARD CARS OR TO EXPRESS COMPANY.

Keeler Seeder, Combined Machine .....................\$9 o

Keeler Seeder, Wheel, Handle and Frame................. 7 on

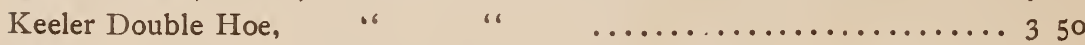

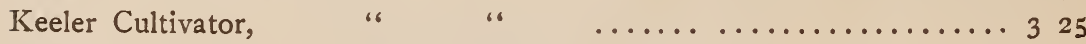

Keeler Plow, " " $"$ " $\quad$ "

Keeler Double Rake, " " $\quad$................. 350

Planet Jr. Combined Seed Drill, Wheel Hoe, Cultivator and Plow ..... 9 oo

Planet Jr. No. 2 Seed Drill........ ................... 7 o

Planet Jr. Double Wheel Hoe, Cultivator and Plow.............. 6 o

Planet Jr. Single Wheel Hoe, Cutivator and Plow............ 450

Firefly Single Wheel Hoe, Cultivator and Plow.............. 375

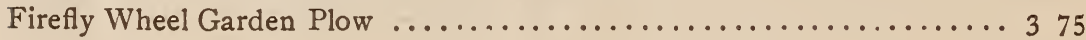

\section{FLOWER SEEDS IN PAPERS.}

Our Flower Seeds are elegantly put up in papers with the price plainly printed thereon. Each paper is ornamented with a fine chromo-lithograph representation of the flower, so that any party not familiar with the flower, can see at a glancea fac simile of the same for purchasing.

For the convenience of those who may not be familiar with the kinds most needed for their trade, would say we have them put in neat trunk-top cases of 100 papers each, in such varieties as our long experience has taught us is most needed by the trade generally. We will furnish any number of papers of each kind that may be ordered separately, or in the cases above mentioned. A list in the form of an order sheet, of the varieties we put up in the above mentioned style, will be forwarded on application.

PRICES.

Boxes of 1.00 papers, valued at $\$ 7.50$, for $\$ 3.00$.

Assorted, per 100 papers, ordered separately, SIXTY PER CENT. off retail price.

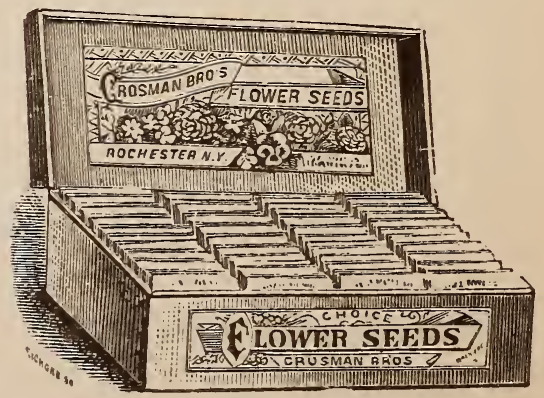




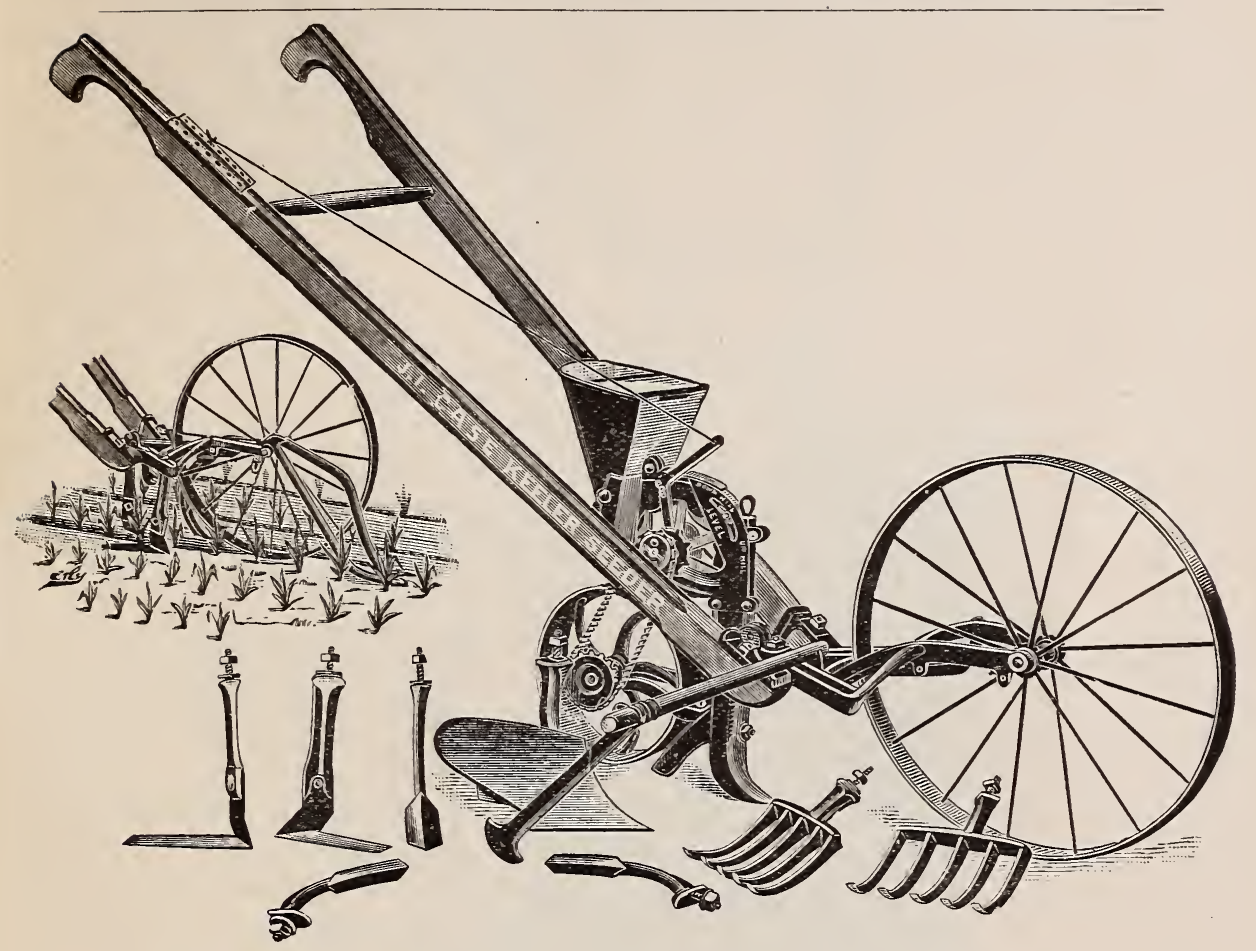

\section{KEELER SEEDER COMBINED MACHINE.}

Retail Price, \$r2.oo.

See wholesale price, page 34 .

A Combined Machine that successfully handles all kinds of grain, either in hills or continuous rous. Thoroughly practical for every tiller of the soil.

The Combined Machine will drill, plant and cultivate any crop until it matures. No other machine required.

It is the only garden hand-drill, planter, cultivator, plow, hoe, grass cutter, weeder, and hiller combined, that will work either in the field or garden without the ground being first specially prepared.

It is the only machine known that will plant corn in the field and drill celery in the garden, and also cover all this range of work without any loose pieces or complicated machinery, and without requiring more than one minute for any adjustment. 


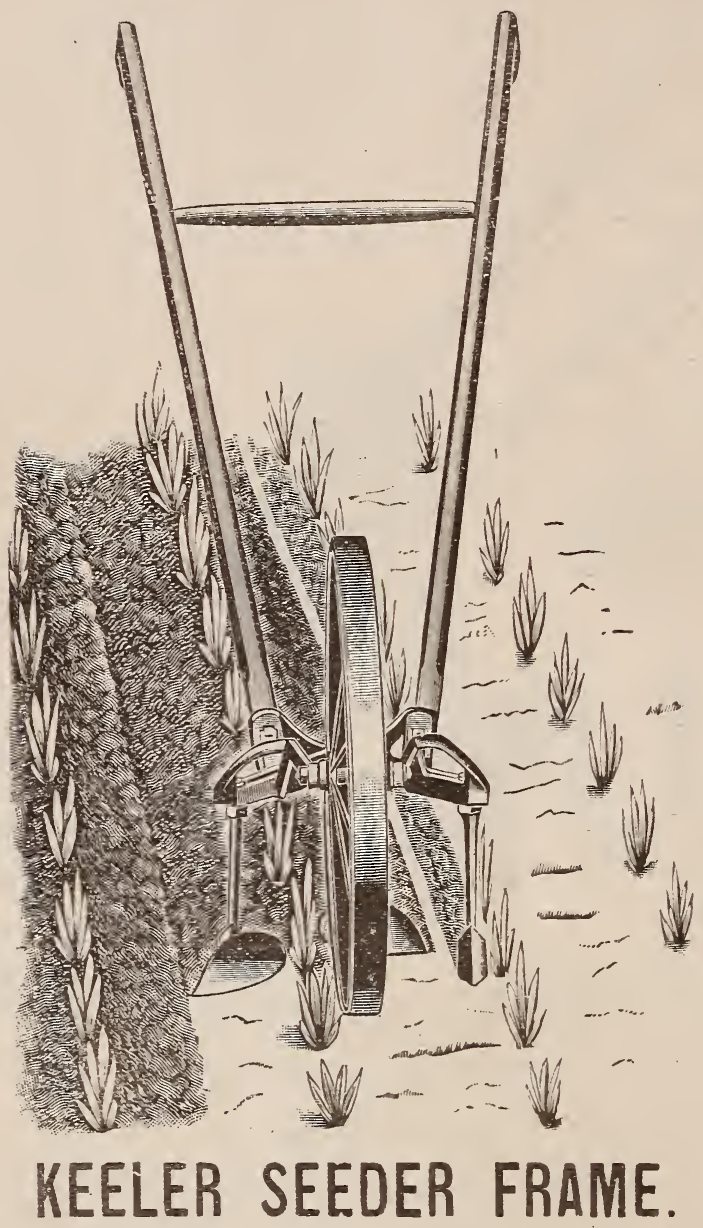

Rigged as a Cultivator. Retail price . . . . ' . . $\$ 4.25$ Rigged as a Plow, throwing soil up to the row or hilling both sides of a row at once . . . . . . . . 4 4.50

This machine is so constructed that it gives the operator great lever of purchasing poiver, and this is the secret of the light draft and the ease of handling of each combination of the Keeler Seeder.

RETAIL PRICE LIST-KEELER MACHINES.

Keeler Seeder, Combined Machine

Keeler Seeder, Wheel, Handle and Frame . . . . . 9.00

Double Hoe

Cultivator

Plow

Double Rake

See wholesale prices, page 34 . 
The "Planet, Jr." Combined Drill, Wheel Hoe, Cultivator, Rake and Plow.

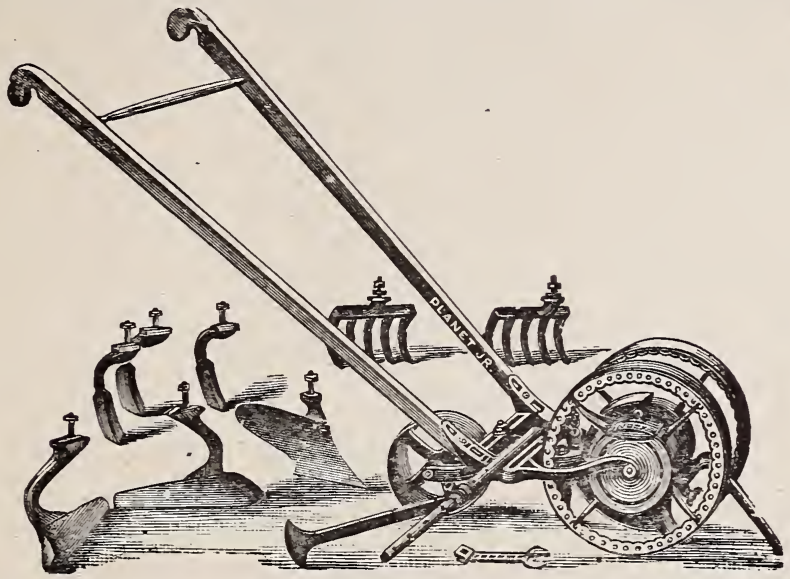

A successful tool, both as a Drill, Wheel Hoe or Cultivator. It is capable of a great variety of operations, and is a thorough success in every way. Retail price, boxed, \$12.00. See wholesale price, page 34 .

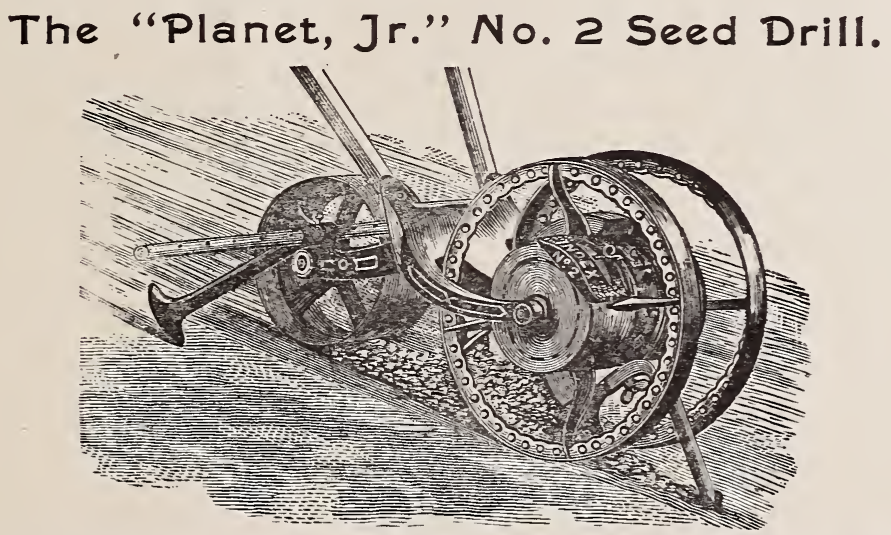

This machine has no Hoe or Cultivator attachments, and can be used as a drill only. In construction and operation it is similar to the Combined Drill, but holds more seed. Retail price, boxed, \$9.00. See wholesale price, page 34 .

The "Planet Jr." Double Wheel Hoe, Cultivator, Rake and Plow.

This tool, as now improved, is perfect in its equipments. A pair of rakes and a pair of leaf lifters have been added to its attachments, which will increase its efficiency. For thorough cultivation of all row crops, this machine has no superior. Retail price, boxed, $\$ 3.00$. See wholesale price, page 34 .

The "Planet Jr." Single Wheel Hoe, Cultivator and Plow.

This deservediy popular tool has also been greatly improved this year. The frame is stronger. lighter and simpler; the wheel broader, to prevent sinking in soft ground, a pair of rakes substituted for an unimportant pair of hoes, and a leaf lifter added without extra expense. Price, boxed for shipment, $\$ 6.00$. See wholesale price, page 34

The "Fire Fly" Single Wheel Hoe, Cultivator and Plow.

This tool has been entirely remodeled, and is now confidently offered as a perfect Wheel Hoe at a low price. Price, boxed, \$5.00. See wholesale price, page 34 . 


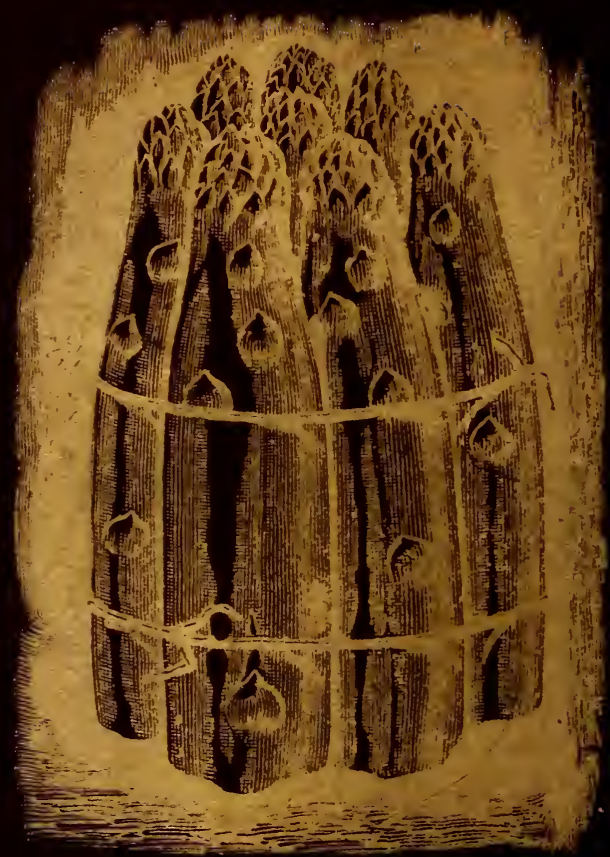

ASPARA\&US-Columbia Manmoth White. see pase 5. 